\title{
LOS COLLETIDAE DE LA SIERRA DE GUADARRAMA (HYMENOPTERA, APOIDEA) ${ }^{1}$
}

\author{
F. J. Ortiz-Sánchez* y C. Ornosa**
}

\begin{abstract}
RESUMEN
Tras el estudio de diversas colecciones entomológicas y la revisión de bibliografía de referencia, los autores estiman que la fauna de colétidos de la Sierra de Guadarrama (centro de la Península Ibérica) está compuesta por 42 especies (31 pertenecientes al género Hylaeus Fabricius, 1793 y 11 al género Colletes Latreille, 1802). Son nuevas para esta sierra un total de 21 especies, y se rechaza la presencia de otras 10 previamente citadas. Desde un punto de vista biogeográfico, los principales elementos son los paleárticos occidentales y los circunmediterráneos (su suma alcanza casi el $50 \%$ del total), siendo relevante el número de endemismos ibéricos (el 16,7\%). Las curvas de fenología alcanzan su máximo en los meses de verano: Hylaeus en julio y agosto y Colletes en agosto.
\end{abstract}

Palabras clave: Colletidae, Sierra de Guadarrama, Península Ibérica.

\section{ABSTRACT}

\section{Colletidae of the Sierra de Guadarrama (Hymenoptera, Apoidea)}

After study of several entomological collections and the reference literature, the authors estimate that the fauna of Colletidae of the Sierra de Guadarrama (central Iberian Peninsula) consists of 42 species (31 belonging to the genus Hylaeus Fabricius, 1793 and 11 to the genus Colletes Latreille, 1802). Twenty-one species are recorded for the first time from these mountains and the presence of ten previously recorded ones is rejected. From a biogeographic point of view, the main elements are West Palaearctic and Circum-Mediterranean (nearly $50 \%$ of the total number). Iberian endemisms represent a relevant portion $(16.7 \%)$. Phenology graphs show a maximum in summer months, with Hylaeus' in July-August and Colletes' in August.

Key words: Colletidae, Sierra de Guadarrama, Iberian Peninsula.

\section{Introducción}

Entre los numerosos estudios entomológicos que sobre la Sierra de Guadarrama se hicieron hace más de dos décadas, e incluso se realizan en la actualidad (Nieves-Aldrey \& Rey del Castillo, 1991; Romera et al., 2002; Romera, 2004), se estudiaron los apoideos, tanto en este entorno (PérezÍnigo, 1980) como a través de una serie de trabajos sobre las distintas familias de esta superfamilia

Subvencionado por el Proyecto CGL2004-04680-C10-05.

* Grupo de Investigación "Transferencia de I+D en el Área de Recursos Naturales". Universidad de Almería. E-04120 La Cañada de San Urbano (Almería), España. E-mail: fjortiz@ual.es

** Departamento de Zoología y Antropología Física. Facultad de Biología. Universidad Complutense. c/ José Antonio Nováis, 2. E-28040 Madrid, España. E-mail: paddy@bio.ucm.es 
(Pérez-Íñigo, 1983, 1984a, 1984b; Ornosa, 1984); uno de los cuales fue dedicado a las familias Colletidae y Halictidae (Pérez-Íñigo, 1984b). En lo referente a los colétidos, este autor relacionó un total de 17 especies pertenecientes al género Hylaeus Fabricius, 1793 y 14 de Colletes Latreille, 1802. Además, aportó numerosos datos sobre su fenología y autoecología.

En tales fechas, esta familia se encontraba muy poco estudiada en la fauna ibérica, dada su complicación taxonómica y el bajo número de especialistas reconocidos entonces. Además, sólo eran de cierta ayuda el trabajo de Dathe (1980) sobre los Hylaeus europeos y, sobre los Colletes de la Paleártica occidental, el de Warncke (1978) o el más antiguo de Noskiewicz (1936), publicaciones que, además, inducían a cometer numerosos errores de identificación, dado que trataban áreas geográficas mucho más amplias que la que nos interesa. Dos publicaciones recientes han venido a cubrir la falta de claves para las especies ibéricas pertenecientes a ambas subfamilias (Ortiz-Sánchez et.al., 2003, 2004).

En el marco del Proyecto Fauna Ibérica, se ha realizado un estudio específico del material de colecciones de la Sierra de Guadarrama, en el que se han revisado, confirmado o corregido las identificaciones existentes. Igualmente, se han estudiado ejemplares adicionales, provenientes de otras colecciones según se relaciona más abajo. El presente trabajo da cuenta de los resultados obtenidos.

\section{Material y Métodos}

Para la caracterización geológica, bioclimática y florística de la Sierra de Guadarrama y la definición de sus ambientes bioclimáticos o pisos de vegetación, así como de la organización estructural de los distintos bosques y demás ecosistemas, pueden consultarse, entre otros, Walter \& Lieth (1960), Costa et al. (1997) y Rivas-Martínez \& Loidi (1999).

Si bien el estudio abarca y se refiere al ámbito de la Sierra de Guadarrama, se ha optado por incluir algún material de localidades adyacentes, tanto por tratarse de zonas de interés (el Monte del Pardo, por ejemplo), como por formar parte integrante, a la vez, del grueso de una de las colecciones que se han revisado (Colección Pérez-Íñigo, UCM, ver más abajo) y que, por lo tanto, aporta más información al estudio realizado.

Se ha revisado el material de la familia Colletidae depositado en las siguientes colecciones: Departamento de Zoología y Antropología Física de la Universidad Complutense de Madrid (UCM), Museo Nacional de Ciencias Naturales de Madrid (MNCN), Servicio de Investigación y Tecnología Agraria de la Junta de Castilla y León (Valladolid, SITA) y datos de los ficheros personales de $\mathrm{H}$. $\mathrm{H}$. Dathe (Hylaeinae; Eberswalde, Alemania) y $\mathrm{M}$. Kuhlmann (Colletinae; Münster, Alemania). Una importante fracción del material del MNCN fue capturada mediante el empleo de una trampa Malaise (José Luis Nieves-Aldrey y Carmen Rey del Castillo leg.).

Se ha seguido, en la presentación de los diferentes taxones, los criterios sistemáticos y sinonímicos reflejados en Ortiz-Sánchez et al. (2002a, 2002b). Por tanto, no se detalla el listado sinonímico para cada especie. Respecto a la validez de la denominación Hylaeus sobre Prosopis Fabricius, 1804 (Warncke, 1972; Pérez-Íñigo, 1984b), véase Dathe (1979a).

Para representar la fenología de esta familia en la Sierra de Guadarrama, se han tenido en cuenta sólo aquellos ejemplares en cuya etiqueta de localidad aparece inequívocamente la fecha de captura. Los datos se han agrupado, por sencillez, por meses.

\section{Resultados}

\section{RELACIÓN DE ESPECIES}

En los Apéndices 1 y 2 se recogen todos los datos de captura de los ejemplares estudiados. En total, suman 1.261 (801 del género Hylaeus y 460 de Colletes). En los que ha sido necesario, se han añadido algunos datos aclaratorios (principalmente, el término municipal) y se han realizado algunas correcciones a partir de Pérez-Íñigo (1983), artículo que se puede consultar también para obtener una mayor información sobre las características de las localidades que ese autor muestreó.

En dichos Apéndices se indica si el material se encontraba identificado previamente, de la siguiente manera:

- Si no se indica nada, los ejemplares no presentaban etiqueta de identificación;

- DATHE y KUHLMANN significa que la información procede de los ficheros de H.H. Dathe y M. Kuhlmann, respectivamente, y los ejemplares están identificados por ellos;

- En los casos en que se menciona una identidad (correcta o no), el identificador es C. PérezIñigo. 
Los datos sobre la distribución de cada especie se han tomado de Ornosa \& Ortiz-Sánchez (2004). Ya que no se había publicado ningún trabajo específico sobre la apoideofauna de la Sierra de Guadarrama antes de los de Pérez-Íñigo, y sólo existían referencias muy aisladas debidas a otros autores, la confirmación o rechazo de citas en esta sierra se basan fundamentalmente en las aportaciones de ese autor.

Familia COLLETIDAE Lepeletier, 1841

Subfamilia HYLAEINAE Viereck, 1916

Género Hylaeus Fabricius, 1793

Subgénero Prosopis Fabricius, 1804

\section{1.- Hylaeus (Prosopis) absolutus (Gribodo, 1894)}

Especie de distribución prácticamente circunmediterránea (falta su confirmación en la parte más oriental de la cuenca mediterránea europea y oriente próximo) más Portugal; presente en las islas mediterráneas de Sicilia y Malta.

Los ejemplares de esta especie en la colección de la UCM estaban incorrectamente identificados como Hylaeus (Prosopis) variegatus (Fabricius, 1798). Es, por tanto, su primera cita en la Sierra de Guadarrama, a pesar de estar bien representada en casi toda la Península Ibérica (hasta el momento no se ha citado en la Cornisa Cantábrica y la mitad norte de Portugal).

\section{2.- Hylaeus (Prosopis) confusus Nylander, 1852}

Especie eurosiberiana (su distribución llega a China y Mongolia y, por el sureste, hasta Turquía y el Cáucaso); confirmada en las islas mediterráneas de Córcega, Cerdeña y Sicilia.

El material citado por Pérez-Íñigo (1984b) como Hylaeus (Prosopis) confusus pertenecía, en realidad, a dos especies distintas. Por tanto, es la primera vez que se cita correctamente de la Sierra de Guadarrama, si bien se encuentra presente en casi toda la Península (escasa de momento en el cuadrante suroccidental).

\section{3.- Hylaeus (Prosopis) convergens Dathe, 2000}

Endemismo ibérico, sólo capturado, de momento, en Picos de Europa, Sierra de Guadarrama y, en Portugal, la región Norte.

El material que se incluye en el Apéndice 1 forma parte de la serie típica (Dathe, 2000).

\section{4.- Hylaeus (Prosopis) coriaceus (Pérez, 1895)}

Especie que se distribuye por la cuenca mediterránea (incluida Creta) y Portugal. Frecuente en toda la Península, aunque de momento no se ha encontrado en Galicia y las islas Baleares.

Se trata de un taxón que ha sido considerado como subespecie de Hylaeus (Prosopis) variegatus hasta hace poco tiempo (por ejemplo, Dathe, 1980). El material depositado en la UCM estaba identificado como esta última. Por tanto, es la primera vez que se cita en la Sierra de Guadarrama en su estatus actualmente reconocido.

\section{5.- Hylaeus (Prosopis) garrulus (Warncke, 1981)}

Endemismo ibérico.

Se cita por primera vez en la Sierra de Guadarrama. Su distribución es, por el momento, mal conocida (Madrid, litoral mediterráneo meridional y, en Portugal, río Duero).

\section{6.- Hylaeus (Prosopis) gibbus S. Saunders, 1850}

Especie probablemente paleártica occidental (en África sólo confirmada en Egipto).

Los ejemplares atribuibles a esta especie y capturados en la Sierra de Guadarrama no pertenecen a la colección de la UCM. Pérez-Íñigo identificó erróneamente como tal algunos individuos de la muy próxima Hylaeus (Prosopis) pictus (Smith, $1853)^{2}$. Por tanto, es la primera cita correcta de esta especie en esta sierra.

En cuanto al resto del área íbero-balear, habita prácticamente en toda España y aún no se ha citado en Portugal.

\section{7.- Hylaeus (Prosopis) pictus (Smith, 1853)}

Repartida por Europa meridional, desde Portugal hasta Grecia; norte de África desde Marruecos hasta Túnez; islas mediterráneas: Baleares, Córcega, Sicilia y Chipre.

Presente en prácticamente toda la Península Ibérica es, sin embargo, su primera cita en la Sierra de Guadarrama (ver la especie anterior).

\section{8.- Hylaeus (Prosopis) praenotatus Förster, 1871}

Especie mediterránea occidental (Francia, España y Marruecos).

El material de la UCM estaba identificado, con dudas, como Hylaeus (Dentigera) rubicola, especie cuya presencia en la Península aún debe ser confir-

${ }^{2}$ De los trabajos de Pérez-Íñigo se extrae que ese autor seguía fielmente a Warncke, quien en 1972 cambia el estatus de $H$. (P.) gibbus, pasándola a subespecie de $H$. (P.) pictus. Por tanto, si Pérez-Íñigo hubiera querido referirse al taxón "pictus" lo habría hecho como Prosopis gibba picta. 
mada. Es su primera cita en España y, por tanto, en la Sierra de Guadarrama ${ }^{3}$.

\section{9.- Hylaeus (Prosopis) signatus (Panzer, 1798)}

Especie paleártica occidental que está representada en nuestra fauna por la siguiente subespecie:

ssp. berlandi (Benoist, 1943)

Subespecie circunmediterránea que llega, por el este, hasta Uzbekistán. En el área íbero-balear, puebla prácticamente toda la Península a excepción, de momento, de la Cornisa Cantábrica; en las islas Baleares ha sido citada de Mallorca.

El único ejemplar de esta especie en la colección de la UCM estaba identificado incorrectamente. Esta hembra, más otros especímenes estudiados en las otras colecciones, suponen la primera cita en la Sierra de Guadarrama.

\section{0.- Hylaeus (Prosopis) variegatus (Fabricius, 1798)}

Especie paleártica occidental, presente en casi toda la Península más la isla de Mallorca.

Una de las especies de Hylaeus más frecuentes en nuestra fauna, lo que coincide en la Sierra de Guadarrama. Había sido identificada correctamente por Pérez-Íñigo (ver también Hylaeus (Prosopis) absolutus e Hylaeus (Prosopis) coriaceus).

\section{Subgénero Dentigera Popov, 1939}

11.- Hylaeus (Dentigera) brevicornis Nylander, 1852

Especie paleártica occidental; presente en las principales islas del Mediterráneo. En el área íberobalear está presente en toda la Península más la isla de Mallorca.

Se confirma en la Sierra de Guadarrama.

\section{2.- Hylaeus (Dentigera) gredleri Förster, 1871}

Especie europea que llega a los Urales y el Cáucaso por el este, pero ausente en el norte del continente; en el Mediterráneo: Baleares, Córcega y Sicilia.

El único ejemplar de esta especie en la colección de la UCM fue identificado por Pérez-Iñigo como la muy próxima Hylaeus (Dentigera) imparilis. Es su primera cita en la Sierra de Guadarrama, a pesar de que en la Península Ibérica está bien representada (a excepción del tercio occidental, donde es muy escasa).

\footnotetext{
${ }^{3}$ A pesar de que Pérez-Íñigo (1984b) cita la especie $P$. stigmorhina Pérez, 1895, sinónima de la que nos ocupa, los ejemplares así identificados pertenecen en realidad a $H$. (P.) pictus.
}

\section{3.- Hylaeus (Dentigera) imparilis Förster, 1871}

Especie circunmediterránea que llega por el norte hasta Hungría, por el oeste hasta Portugal y por el este hasta el Cáucaso; está presente además en todas las islas grandes del Mediterráneo (Mallorca y Menorca incluidas). Habita toda la Península.

Algunos ejemplares de la colección de la UCM estaban bien identificados, aunque otros corresponden en realidad a otras especies (ver Apéndice 1). Se confirma, pues, en la Sierra de Guadarrama.

\section{4.- Hylaeus (Dentigera) penalaris Dathe, 1979}

Endemismo ibérico sólo capturado en la Sierra de Guadarrama, Badajoz y algunas localidades del tercio norte de Portugal próximas al Duero.

En la colección de la UCM no existe ningún ejemplar de esta especie, a pesar de la coincidencia temporal de los muestreos de Pérez-Íñigo con aquéllos en que Ebmer recolectó los ejemplares de la serie típica (ver Dathe, 1979b). Tampoco existen especímenes atribuibles a ella en las otras colecciones estudiadas.

\section{Subgénero Paraprosopis Popov, 1939}

\section{5.- Hylaeus (Paraprosopis) clypearis (Schenck, 1853)}

Presente en casi toda Europa (por el norte hasta Dinamarca, por el este hasta Ucrania), norte de África, desde Marruecos al norte de Libia, y todas las grandes islas mediterráneas (incluyendo Mallorca y, con dudas, Ibiza). Sus citas en la Península Ibérica cubren todo el territorio.

Los machos estudiados por Pérez-Iñigo estaban correctamente identificados; sin embargo, este autor adjudicó este nombre a hembras que, en realidad, pertenecen a otras especies. Se confirma en la Sierra de Guadarrama.

\section{6.- Hylaeus (Paraprosopis) lineolatus (Schenck, 1861)}

Especie mediterránea, que penetra hasta Centroeuropa (Alemania) y Rusia y llega al Cáucaso, Irán e Israel; en el norte de África sólo se ha citado en Argelia; en las islas mediterráneas de Creta y Chipre se han descrito sendas subespecies endémicas. En el área íbero-balear falta en el tercio occidental y las islas Baleares.

A excepción de un macho, todo el material estaba identificado correctamente. Se confirma en la Sierra de Guadarrama.

\section{7.- Hylaeus (Paraprosopis) pictipes Nylander, 1852}

Especie paleártica occidental; ha sido encontrada en toda Europa (incluyendo Gran Bretaña) hasta 
el Cáucaso, Oriente Próximo y todo el norte de África, a excepción de Egipto.

Pérez-Íñigo confundió esta especie con Hylaeus (Hylaeus) communis. De hecho, el material que realmente pertenece a la que nos ocupa y que aquí se cita no fue estudiado por ese autor. Es la primera cita correcta para la Sierra de Guadarrama, si bien ya se conocía prácticamente en toda la Península Ibérica más la isla de Mallorca.

18.- Hylaeus (Paraprosopis) sinuatus (Schenck, 1853)

Presente en toda Europa, hasta el Cáucaso, Irán, Iraq e Israel; en el norte de África: de Marruecos a Túnez. En el área íbero-balear está presente en provincias dispersas que cubren gran parte de la Península, a excepción de Galicia y provincias limítrofes.

Es su primera cita en la Sierra de Guadarrama.

\section{9.- Hylaeus (Paraprosopis) soror (Pérez, 1903)}

Europa occidental y meridional, alcanzando aisladamente Centroeuropa, hasta Irán; en el Mediterráneo, islas de Córcega, Cerdeña, Sicilia y Chipre.

Se cita por primera vez en la Sierra de Guadarrama. Con esta cita, su distribución conocida en la Península ocupa provincias dispersas: Huesca, Barcelona, Madrid, Granada, Málaga, Cádiz y Algarve.

\section{Subgénero Hylaeus Fabricius, 1793}

\section{0.- Hylaeus (Hylaeus) angustatus (Schenck, 1861)}

Especie paleártica; de las islas mediterráneas, ha sido citada de Córcega, Sicilia y Creta.

El material de la UCM estaba identificado erróneamente: la mayoría de ejemplares como Hylaeus (Prosopis) confusus, más una hembra, como Hylaeus (Dentigera) brevicornis.

Se cita por primera vez en la Sierra de Guadarrama, aunque está presente en casi toda la Península, a excepción del cuadrante suroccidental.

\section{1.- Hylaeus (Hylaeus) communis Nylander, 1852}

Ampliamente distribuida por toda Europa, alcanza el Cáucaso, Turquía, Irán e Iraq, Kazajstán, hasta Siberia; en África sólo se ha mencionado en Marruecos; presente en las islas de Córcega, Cerdeña, Sicilia y Creta.

En la colección de la UCM existen varios ejemplares de esta especie, aunque fueron erróneamente identificados. Se desconocía en la Sierra de Guadarrama. En nuestra Península es una especie que se encuentra bien representada, pero aún no ha sido citada en el cuadrante suroccidental.

\section{2.- Hylaeus (Hylaeus) difformis (Eversmann, 1852)}

Especie muy ampliamente distribuida por la región paleártica occidental, que está representada en nuestro territorio por la subespecie:

ssp. hispanicus (Warncke, 1972)

Presente en la Península Ibérica y Marruecos. En el área íbero-balear está bien representada en la mitad oriental de la Península, siendo Ávila y Cádiz las provincias más occidentales de donde se ha citado; citada en Portugal, pero sin localidades concretas.

Se confirma en la Sierra de Guadarrama, si bien había sido citada sin especificar la subespecie (Pérez-Íñigo, 1984b).

\section{3.- Hylaeus (Hylaeus) gracilicornis (Morawitz, 1867)}

Especie con una amplia distribución eurosiberiana.

El material que se cita en el Apéndice 1 constituye el primer registro para la Sierra de Guadarrama, siendo una especie muy escasa en el área íbero-balear: sólo había sido citada previamente en la Sierra de Gádor (Almería).

\section{4.- Hylaeus (Hylaeus) ibericus Dathe, 2000}

Endemismo ibérico, recolectado en las sierras de la Demanda, Ezcaray, Guadarrama y Nevada (Dathe, 2000).

El estudio de las colecciones mencionadas no ha aportado ningún dato nuevo sobre su distribución en Guadarrama, al no existir ejemplares atribuibles a esta especie. Al igual que en el caso de otro endemismo, Hylaeus (Dentigera) penalaris, se da la curiosidad de que Pérez-Iñigo muestreó en idénticas fechas una localidad donde Ebmer capturó el material que se cita en la descripción de la especie (Dathe, 2000).

\section{5.- Hylaeus (Hylaeus) nigrita (Fabricius, 1798)}

Presente en toda Europa (aunque no hay citas concretas en Portugal, por ejemplo), llegando, por Turquía, al Cáucaso e Irán; citada también en China (ver Celary \& Dylewska, 1988).

Todos los ejemplares de la UCM estaban identificados correctamente. Se confirma, pues, su presencia en la Sierra de Guadarrama. En el área íbero-balear se halla bien representada en la mitad oriental, más las provincias de Zamora, Salamanca y Sevilla. 
Subgénero Lambdopsis Popov, 1939

26.- Hylaeus (Lambdopsis) annularis (Kirby, 1802)

Especie probablemente paleártica occidental (aún no se ha citado de las áreas más orientales de esta distribución), presente en la isla de Córcega.

Todos los ejemplares recogidos en el Apéndice 1 proceden de colecciones diferentes a la que estudió Pérez-Íñigo (pero ver la especie siguiente). Es nueva para la Sierra de Guadarrama. En el área íbero-balear, además de la Sierra de Guadarrama, cuenta con pocas citas pero que abarcan casi todo el territorio.

27.- Hylaeus (Lambdopsis) crassanus (Warncke, 1972)

Citada en Europa meridional y Suiza; según Warncke (1992), existe una población disyunta en el Sáhara argelino.

Casi todos los ejemplares que existen en la UCM correspondientes a esta especie estaban identificados como "Prosopis annulata (Linnaeus, 1758)". Ya que, en realidad, Hylaeus (Hylaeus) annulatus (L.) es una especie bastante distinta de la que nos ocupa, cuya presencia en la fauna íbero-balear no ha sido confirmada (Ornosa \& Ortiz-Sánchez, 2004), podría ser que Pérez-Íñigo identificara originalmente el material como la muy próxima Hylaeus (Lambdopsis) annularis y, a la hora de transcribir los datos, cometiera el error. Era, por tanto, desconocida hasta ahora en la Sierra de Guadarrama.

Con esta cita, su distribución conocida en el área íbero-balear es el centro de la Península (Salamanca, Sierra de Guadarrama y alrededores) y el tercio meridional de Portugal.

\section{Subgénero Koptogaster Alfken, 1912}

28.- Hylaeus (Koptogaster) punctulatissimus Smith, 1842

Presente en toda Europa, Sicilia, Turquía, llegando al Cáucaso e Irán; en Marruecos existe una población relicta en el Alto Atlas (Warncke, 1992).

El material que se ha estudiado de esta especie fue capturado con trampa Malaise y es, de momento, muy escaso. Se trata de la primera cita en la Sierra de Guadarrama. Por tanto, su distribución conocida en el área íbero-balear es: Cataluña, Valladolid y las sierras de Guadarrama, María y Nevada; además, en las regiones Norte y Centro de Portugal.

\section{Subgénero Abrupta Méhely, 1935}

\section{9.- Hylaeus (Abrupta) cornutus Curtis, 1831}

Especie de amplia distribución, probablemente paleártica occidental (aún no citada de Rusia); presente en las grandes islas mediterráneas.
Se trata de un taxón muy característico, que se cita por primera vez para la Sierra de Guadarrama. Con esta aportación, su distribución en el área íbero-balear comprende las provincias españolas de Barcelona, León, Zamora, Salamanca, Valladolid, Segovia, Madrid, Zaragoza, Cádiz, Sevilla, Granada y Málaga; en Portugal: Oporto; en las islas Baleares: Mallorca.

\section{Subgénero Spatulariella Popov, 1939}

30.- Hylaeus (Spatulariella) hyalinatus Smith, 1842

Especie de morfología variable, ampliamente distribuida por toda Europa (incluyendo a Gran Bretaña), Turquía, Irán y el Cáucaso. Presente en islas del Mediterráneo tales como Córcega, Cerdeña, Sicilia y Creta. Introducida en Estados Unidos (Ascher, 2001). Presenta una serie de subespecies que se corresponden con poblaciones que quedan más o menos aisladas geográficamente, entre las que se encuentra la que habita en el área íbero-balear.

ssp. aragonensis (Pittioni, 1950)

Es un endemismo presente en toda la Península Ibérica más la isla de Mallorca.

Ya había sido recogida por Pérez-Ínigo (1984b), aunque no especificó la subespecie. Por tanto, si bien la especie estaba confirmada en Guadarrama, se cita por primera vez esta población.

31.- Hylaeus (Spatulariella) sulphuripes (Gribodo, 1894)

Bien representada en el norte de África, desde Marruecos a Turquía, además de Francia y la Península Ibérica; especie introducida en las islas Canarias (Dathe, 1993). En el área íbero-balear está bien representada en las provincias mediterráneas y los Pirineos, Lisboa y algunas provincias aisladas en el centro de la Península (Burgos, Salamanca).

Ésta es su primera cita para la Sierra de Guadarrama.

Subfamilia COLLETINAE Lepeletier, 1841

Género Colletes Latreille, 1802

\section{Grupo nigricans}

\section{2.- Colletes eous Morice, 1904}

Ampliamente distribuida en la región paleártica, llegando al suroeste de Siberia, Turkestán, Irán, Asia Menor, Cáucaso y toda la cuenca mediterránea.

Se confirma en la Sierra de Guadarrama, con lo que su distribución conocida en el área íbero-balear 
no sufre alteración (Galicia, Pirineos centrales, Zaragoza, Sierra de Guadarrama, Sierra Nevada, Granada y Murcia; en Portugal: Serras da Estrela y da Lousã).

\section{3.- Colletes nigricans Gistel, 1857}

Presente en Europa meridional (desde Portugal a la antigua Yugoslavia, incluyendo el sur de Francia, Suiza y Alemania), norte de África (Marruecos, Argelia, Sáhara occidental) y la isla de Sicilia.

A excepción de una hembra, identificada como Colletes kozlovi Friese, $1913^{4}$, todos los ejemplares de la UCM estaban correctamente etiquetados. Se confirma en la Sierra de Guadarrama, con lo que no se amplía su distribución conocida en nuestra fauna (bien representada en Portugal y, en España, abunda en la franja litoral este y sur, con capturas aisladas en Aragón, Submeseta Norte, Sierra de Guadarrama, Madrid, Toledo y Ciudad Real).

\section{Grupo hylaeiformis}

34.- Colletes hylaeiformis Eversmann, 1852

Distribución muy amplia, extendiéndose por Europa meridional y central, desde Turkmenistán y el sur de Rusia hasta la Península Ibérica y, por el norte, hasta Polonia.

Especie muy característica; algunos ejemplares de la UCM estaban bien identificados, los demás como C. eous o $C$. succinctus. Se confirma en la Sierra de Guadarrama, por lo que no supone una ampliación de su distribución conocida en la Península (Submeseta Norte, centro y cuadrante nororiental de la Península, Sierra Nevada y Alicante; en Portugal, tercio central).

\section{5.- Colletes ligatus Erichson, 1835}

Especie mediterránea occidental (sur de Francia, Península Ibérica, Marruecos, Argelia, Túnez).

Esta especie, muy próxima a la anterior, fue confundida por Pérez-Ĩnigo, sin embargo, con Colletes maidli Noskiewicz, 1936. Es la primera vez que se cita en la Sierra de Guadarrama, por lo que su distribución en nuestra área se amplía al centro de la Península, estando presente en las provincias del litoral español este y sur, además de la Sierra de Gadarrama y, en Portugal, las regiones centro y norte.

\footnotetext{
${ }^{4}$ C. kozlovi es una especie que hasta ahora ha sido capturada muy raras veces, y está distribuida por Mongolia y el Turkestán (Noskiewicz, 1936; Kuhlmann, com. pers.).
}

\section{Grupo fodiens}

\section{6.- Colletes abeillei Pérez, 1903}

Especie mediterránea occidental, citada en Italia, Francia, España, Portugal y Marruecos, así como en Sicilia y las islas Baleares. En el área íbero-balear está presente en toda España (menos Galicia y provincias limítrofes), el tercio central de Portugal y, en las islas Baleares, Mallorca y Menorca.

La mayoría de los ejemplares de la UCM estaban correctamente identificados, a excepción de algunos que estaban etiquetados como Colletes mlokossewiczi Radoszkowski, 1891 (ver más abajo). Se confirma en la Sierra de Guadarrama, por lo que su distribución conocida no varía.

\section{7.- Colletes fodiens (Geoffroy, 1785)}

Especie paleártica occidental que está representada en nuestra fauna por la subespecie:

ssp. hispanicus Noskiewicz, 1936

Subespecie ibérica, presente prácticamente en toda la Península, menos frecuente en el tercio sur y ausente, por el momento, en las islas Baleares.

Se trata de uno de los taxones más frecuentes en la Península Ibérica y el más abundante en las colecciones estudiadas. Sin embargo, la mayoría del material estaba identificado y publicado (Pérez-Iñigo, 1984b) como Colletes albescens Noskiewicz, 1936 (sinónima de Colletes noskiewiczi Cockerell, 1942, especie muy próxima a la identificación correcta) y algunos ejemplares como Colletes succinctus (Linnaeus, 1758). Este material, por tanto, representa su primera cita para la Sierra de Guadarrama, si bien su distribución en el área íbero-balear no varía.

\section{8.- Colletes noskiewiczi Cockerell, 1942}

Endemismo ibérico, presente en Gerona, Guadalajara, Sierra Nevada y Sierra de Gádor.

El único ejemplar de esta especie en la UCM, estaba identificado como Colletes daviesanus Smith, 1846 con dudas. Es, por tanto, la primera cita para esta especie en la Sierra de Guadarrama, con lo que se amplía su distribución conocida.

\section{9.- Colletes similis Schenck, 1853}

Especie paleártica, presente prácticamente en toda la España peninsular, a excepción del cuadrante suroccidental; ausente de momento en Portugal y las islas Baleares.

A pesar de existir varios ejemplares en la UCM, no estaban identificados correctamente. Se cita por primera vez en la Sierra de Guadarrama. 
Tabla 1.- Composición biogeográfica de la fauna de Colletidae de la Sierra de Guadarrama (centro de la Península Ibérica).

Tabla 1.- Biogeographic composition of the Colletidae fauna of the Sierra de Guadarrama (central Iberian Peninsula).

\begin{tabular}{lccc}
\hline & Hylaeus & Colletes & Total \\
\hline Elementos paleárticos & 1 & 3 & $4(9,5 \%)$ \\
Elementos paleárticos occidentales & 10 & - & $10(23,8 \%)$ \\
Elementos eurosiberianos & 3 & - & $3(7,1 \%)$ \\
Elementos europeos & 2 & 1 & $3(7,1 \%)$ \\
Elementos europeos meridionales & 1 & 1 & $2(4,8 \%)$ \\
Elementos circunmediterráneos & 7 & 2 & $9(21,4 \%)$ \\
Elementos mediterráneos occidentales & 2 & 2 & $4(9,5 \%)$ \\
Endemismos ibéricos & 5 & 2 & $7(16,7 \%)$ \\
TOTAL & $\mathbf{3 1}$ & $\mathbf{1 1}$ & $\mathbf{4 2}$
\end{tabular}

\section{Grupo senilis}

40.- Colletes mlokossewiczi Radoszkowski, 1891

Especie presente en toda Europa meridional, del sur de los Urales a Portugal, incluyendo además Asia Menor, Irán, Uzbekistán, Kazajstán y el Cáucaso; en el Mediterráneo, islas de Córcega y Sicilia. En la Península Ibérica se ha citado en Coimbra, Beja (Portugal), Zamora, Cuenca, Madrid (Sierra de Guadarrama) y Valladolid (España).

Citada por Pérez-Íñigo (1984b) a partir de cuatro hembras que había identificado con dudas y que, en realidad, pertenecen a Colletes abeillei (ver más arriba). Dicho autor sólo estudió una hembra de esta especie, pero no la identificó como tal. Se confirma en la Sierra de Guadarrama.

\section{Grupo succinctus}

\section{1.- Colletes succinctus (Linnaeus, 1758)}

Especie paleártica, ampliamente repartida. Bien representada en toda la Península (peor en el cuadrante noroccidental) e islas Baleares.

Citada erróneamente (Pérez-Íñigo, 1984b), por medio de ejemplares que corresponden a Colletes hylaeiformis. El único individuo de Colletes succinctus que estudió Pérez-Iñigo en la UCM lo identificó como la muy próxima Colletes collaris Dours, 1872. Por tanto, es la primera cita correcta para la Sierra de Guadarrama.

\section{Grupo albomaculatus}

42.- Colletes albomaculatus (Lucas, 1849)

Circunmediterránea, llegando hasta el Cáucaso; presente en las islas Baleares, Cerdeña, Sicilia y Creta. Bien representada en toda la Península
Ibérica, aunque menos frecuente en el cuadrante noroccidental; en las islas Baleares: Mallorca.

Los ejemplares de la UCM estaban bien identificados ${ }^{5}$. Se confirma, pues, en la Sierra de Guadarrama.

\section{CONSIDERACIONES ZOOGEOGRÁFICAS}

La Tabla 1 presenta la composición de la fauna de Colletidae de la Sierra de Guadarrama desde el punto de vista zoogeográfico. Dadas las condiciones de la sierra, su composición fitogeográfica (Rivas-Martínez \& Loidi, 1999), y coincidiendo con otros grupos de insectos (por ejemplo, Viejo \& Templado, 1986; Romera, 2004), los elementos principales que la componen son los paleárticos occidentales y los circunmediterráneos, alcanzando entre ambos casi la mitad de las especies. Destaca asimismo el alto número de endemismos ibéricos, con un total de siete (el $16,7 \%$ del total).

\section{FenOlogía}

En las figuras 1 y 2 se dibujan las gráficas correspondientes a la fenología de la familia Colletidae, tanto en conjunto como por géneros. Se desprende inmediatamente la condición de familia típica de los meses de verano, tal como ya han indicado autores como Ortiz-Sánchez \& Aguirre-Segura (1991), Kuhlmann (1996), Comba \& Comba (2001), etc., con las consecuentes acomodaciones a los ecosistemas de montaña. La regla general en los Apoidea es que la aparición de los machos suele ser

${ }^{5}$ Aunque como su sinónima Colletes spectabilis Morawitz, 1868. 

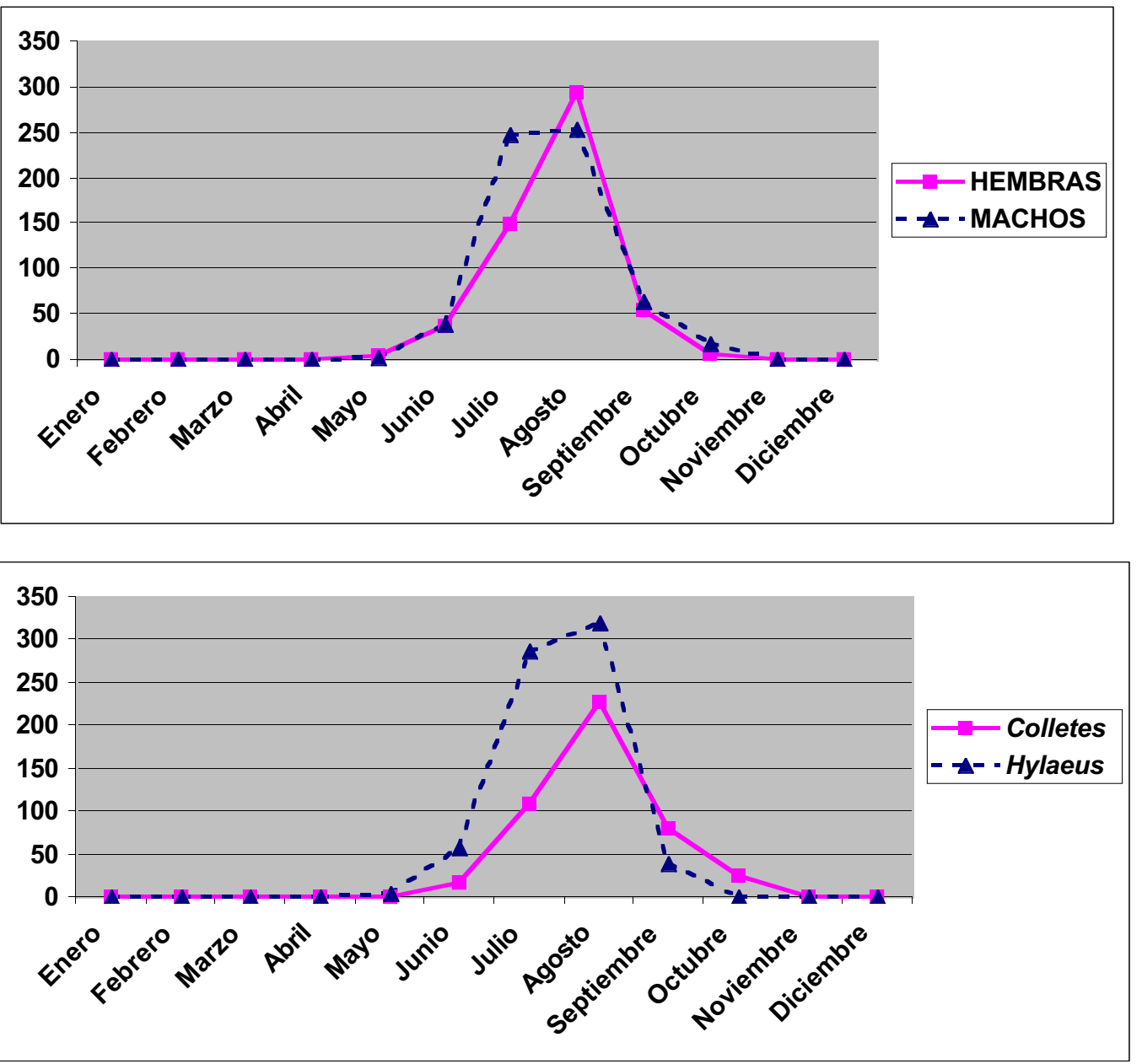

Fig. 1.- Curvas de fenología de la familia Colletidae en la Sierra de Guadarrama (centro de la Península Ibérica).

Fig. 1.- Phenology charts of the family Colletidae in the Sierra de Guadarrama (central Iberian Peninsula).

anterior a la de las hembras. En este sentido, se observa que los machos alcanzan sus valores máximos antes que las hembras, más que una aparición en el campo más prematura. En cuanto a la curva según los géneros, Hylaeus centra su ciclo en los meses de julio y agosto, mientras que Colletes es algo más tardío, con el máximo de actividad en agosto. El máximo de Hylaeus es más amplio, ocupando dos meses, lo que se debe en gran medida a la explosión de machos capturados mediante trampas Malaise en el mes de agosto (principalmente de las especies Hylaeus (Dentigera) gredleri, Hylaeus (Paraprosopis) clypearis, o Hylaeus (Hylaeus) communis).

\section{Discusión}

El estudio realizado revela la existencia de 17 especies de Hylaeus en la UCM. Este número es igual al reflejado por Pérez-Íñigo (1980, 1984b), pero no concuerdan las especies una a una, ya que algunas fueron incorrectamente identificadas y los ejemplares adjudicados a otras que, una vez revisadas, no se puede afirmar que se presenten en la Sierra de Guadarrama. Éste es el caso de Hylaeus (Prosopis) duckei (Alfken, 1904); Hylaeus (Dentigera) rubicola S. Saunders, 1850; Hylaeus (Paraprosopis) styriacus Förster, 1871; Hylaeus (Hylaeus) annulatus (Linnaeus, 1758) y "Prosopis 

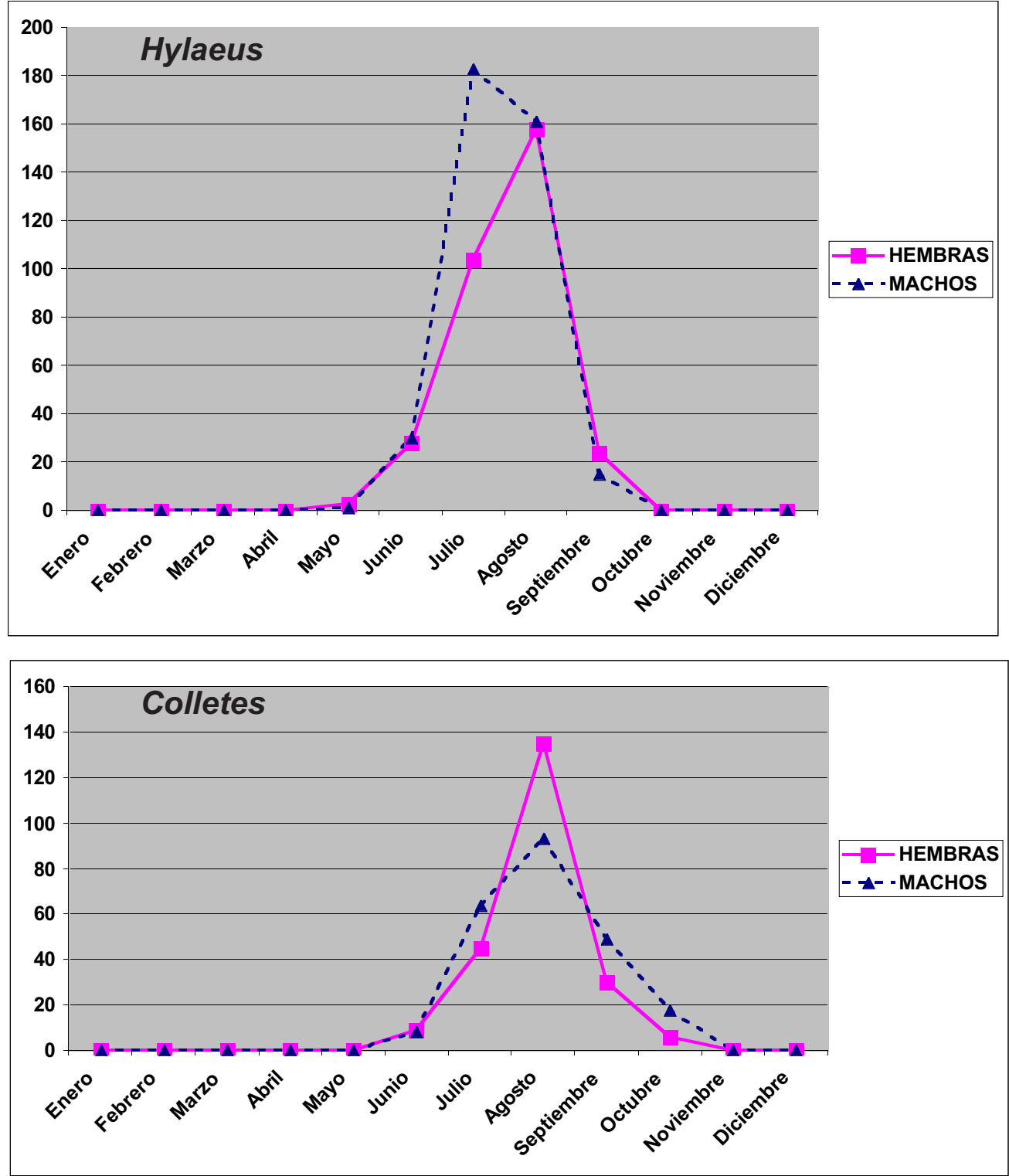

Fig. 2.- Curvas de fenología de los géneros Hylaeus y Colletes en la Sierra de Guadarrama (centro de la Península Ibérica).

Fig. 2.- Phenology charts of the genus Hylaeus and Colletes in the Sierra de Guadarrama (central Iberian Peninsula).

minuta (Fabricius, 1798)"', 7 . Por otro lado, hay una serie de especies que se añaden a la lista de ese autor, ya que los ejemplares fueron adjudicados a otras:

${ }^{6}$ Hylaeus minutus Fabricius, 1798 es en realidad un halíctido, Lasioglossum (Evylaeus) minutum (Fabricius, 1798), descrito con un macho de las Indias Occidentales (S. Thomas; Ebmer, 1974) y Apis minuta Fabricius, 1793 (non Schrank, 1781) es nomen dubium, aunque tradicionalmente se la coloca en la lista sinonímica de Hylaeus (Dentigera) sinuatus.
Hylaeus (Prosopis) absolutus, Hylaeus (Prosopis) coriaceus, Hylaeus (Prosopis) praenotatus, Hylaeus (Prosopis) signatus, Hylaeus (Dentigera) gredleri,

${ }^{7}$ De hecho, las especies Hylaeus (Prosopis) duckei, Hylaeus (Paraprosopis) styriacus e Hylaeus (Hylaeus) annulatus no existen siquiera en la fauna ibérica (Ornosa \& Ortiz-Sánchez, 2004). 
Hylaeus (Hylaeus) angustatus, Hylaeus (Hylaeus) communis e Hylaeus (Lambdopsis) crassanus. Finalmente, hay unas especies que se añaden gracias al estudio de otras colecciones o a la comunicación por parte de otros especialistas: Hylaeus (Prosopis) convergens (cuya serie típica procede de la Sierra de Guadarrama; ver Dathe, 2000), Hylaeus (Prosopis) garrulus (descrita con material de la provincia de Alicante; ver Warncke, 1981), Hylaeus (Paraprosopis) pictipes (que fue citada por Pérez-Íñigo pero basada en identificaciones incorrectas), Hylaeus (Paraprosopis) sinuatus, Hylaeus (Paraprosopis) soror, Hylaeus (Hylaeus) gracilicornis, Hylaeus (Lambdopsis) annularis, Hylaeus (Koptogaster) punctulatissimus e Hylaeus (Spatulariella) sulphuripes. Además, y aunque no se han estudiado ejemplares, destacan Hylaeus (Dentigera) penalaris, que fue también descrita con material procedente de esta sierra (Dathe, 1979b), e Hylaeus (Hylaeus) ibericus, especie descrita recientemente, cuya serie típica procede de Sierra Nevada y también aparece en Guadarrama (Dathe, 2000).

En cuanto al género Colletes, se relacionaban 14 especies (Pérez-Iñigo, 1980, 1984b) pero, en realidad, son sólo 11. Hay que eliminar de la lista aportada por dicho autor las siguientes: Colletes merceti, Colletes acutus, Colletes collaris, Colletes daviesanus (especie no confirmada de momento en la Península según Ornosa \& Ortiz-Sánchez, 2004), Colletes kozlovi y Colletes maidli.

Otras especies son citadas por primera vez de esta sierra a partir de material de la UCM cuya identificación previa era errónea: Colletes ligatus, Colletes fodiens, Colletes noskiewiczi y Colletes similis. Finalmente, el estudio de las otras colecciones no aporta especies adicionales a la lista.

\section{Conclusiones}

Con 31 especies del género Hylaeus, que representan el $63,3 \%$ respecto al total de la fauna ibérica, y 11 de Colletes (el 36,7\%), la Sierra de Guadarrama, por su extensión, gradiente altitudinal y variedad florística, representa un enclave privilegiado en cuanto a su fauna de abejas de la familia Colletidae.

\section{AGRADECIMIENTOS}

Los autores quieren mostrar su agradecimiento a las instituciones que han permitido estudiar sus colecciones entomológicas, relacionadas en el apartado de Material y Métodos. Igualmente, se agradece a H. H. Dathe y M. Kuhlmann (espe- cialistas en Hylaeinae y Colletinae, respectivamente) por su valiosa ayuda en la identificación de numerosos ejemplares, además de por haber aportado algunos datos de captura de ejemplares de esta familia en la Sierra de Guadarrama. Por último, es obligado reconocer el importante trabajo realizado por Carlos Pérez-Íñigo, porque él abrió el camino para que éste fuera posible.

\section{Referencias}

Ascher, J. S., 2001. Hylaeus hyalinatus Smith, a European bee new to North America, with notes on other adventive bees (Hymenoptera: Apoidea). Proceedings of the Entomological Society of Washington, 103(1): 184-190.

Costa, M., Morla, C. \& Sainz, H., 1997. Los bosques ibéricos. Planeta. Barcelona. 576 pp.

Celary, W. \& Dylewska, M., 1988. Colletidae (Hymenoptera, Apoidea) Polski. Polskie Pismo Entomologiczne, 58: 359-382.

CombA, M. \& ComBA, L., 2001. Gli Apoidei (Hymenoptera: Aculeata) alpini: diversità e abbondanza in alcune fasce altitudinali delle Valli del Pellice, Angrogna e Germanasca (Alpi Cozie). Bollettino del Museo regionale di Scienze naturali di Torino, 18(1): 11-97.

Dathe, H. H., 1979a. Der Gattungsname der Maskenbienen: Hylaeus versus Prosopis. Linzer biologische Beiträge, 11: 147-154.

DAthe, H. H., 1979b. Zum Vorkommen von Hylaeus F.Arten im Gebirge nebst Festlegung von Lectotypen. Linzer biologische Beiträge, 11: 155-168.

DAthe, H. H., 1980. Die Arten der Gattung Hylaeus F. in Europa (Hymenoptera: Apoidea, Colletidae). Mitteilungen aus dem Zoologischen Museum in Berlin, 56(2): 207-294.

DAthe, H. H., 1993. Taxonomie und Verbreitung der Gattung Hylaeus F. auf den Kanarischen Inseln (Insecta: Hymenoptera: Apoidea: Colletidae). Veröffentlichungen Übersee-Museum Bremen (Naturwissenschaftlichen), 12: 743-760.

Dathe, H. H., 2000. Studien zur Systematik und Taxonomie der Gattung Hylaeus F. (3). Revision der Hylaeus-nivalis-Gruppe in Europa und Klärung weiterer westpaläarktischer Arten (Apidae, Colletinae). Beiträge zur Entomologie, 50(1): 151-174.

EBmer, A. W., 1974. Von Linné bis Fabricius beschriebene westpaläarktische Arten der Genera Halictus und Lasioglossum (Halictidae, Apoidea). Nachrichtenblatt der Bayerischen Entomologen, 23 (6): 111-127.

Kuhlmann, M., 1996. Contribution to the knowledge of the bee and wasp fauna (Hymenoptera Aculeata) of the Serra da Estrela, Portugal. Boletim da Sociedade portuguesa de Entomologia, 166(VI-16): 213-228. 
Nieves-Aldrey, J. L. \& Rey del Castillo, C., 1991. Ensayo preliminar sobre la captura de insectos por medio de una trampa «Malaise» en la Sierra de Guadarrama (España) con especial referencia a los himenópteros (Insecta, Hymenoptera). Ecología, 5: 383-403.

Noskiewicz, J., 1936. Die paläarktischen ColletesArten. Prace Naukowe; Wydawnictwo Towarzystwa Naukowego we Lwowie, (2) 3: 1-532, 28 taf.

Ornosa, C., 1984. La subfamilia Bombinae (Hym., Apidae) de la fauna española. Ed. Universidad Complutense de Madrid. Colección Tesis Doctorales, $n^{\circ}$ 203/84: vii + 334 pp.

Ornosa, C. \& OrTIZ-SÁnchez, F. J., 2004. Hymenoptera, Apoidea I. En: Fauna Ibérica, vol. 23. Ramos, M.A. et al., (eds.). Museo Nacional de Ciencias Naturales. CSIC. Madrid. 556 pp.

Ortiz-SÁnchez, F. J. \& Aguirre-SegurA, A., 1991. Estructura y dinámica estacional de una comunidad de Apoidea (Hymenoptera) en Almería. Eos, 67: 3-22.

Ortiz-SÁnchez, F. J., Ornosa, C \& DAThe, H. H., 2002a. Catálogo sinonímico de los Colletidae ibéricos. I, subfamilia Hylaeinae (Hymenoptera, Apoidea). Entomofauna, 23(21): 249-266.

Ortiz-SÁnchez, F. J., Ornosa, C. \& Dathe, H. H., 2003. Claves de identificación para las especies ibéricas del género Hylaeus Fabricius, 1793 (Hymenoptera, Colletidae). Boletín de la Asociación española de Entomología, 27(1-4): 169-207.

Ortiz-SÁnchez, F. J., Ornosa, C. \& Kuhlmann, M., 2002b. Catálogo sinonímico de los Colletidae ibéricos. II, subfamilia Colletinae (Hymenoptera, Apoidea). Entomofauna, 23(22): 267-278.

Ortiz-Sánchez, F. J., Ornosa, C. \& Kuhlmann, M., 2004. Claves de identificación para las especies ibéricas del género Colletes Latreille, 1802 (Hymenoptera, Colletidae). Zoologica baetica, 15: 3-37.

Pérez-Íñigo, C., 1980. Los Ápidos (Hym., Apoidea) de la Sierra de Guadarrama. Tesis Doctoral. Universidad Complutense de Madrid. Madrid. 417 pp.

PÉrez-ÍñIgo, C., 1983. Los Ápidos de la Sierra de Guadarrama I. Familias Melittidae y Anthophoridae (Hymenoptera, Apoidea). Graellsia, 38 [1982]: 43-65.

PÉreZ-ÍñIgO, C., 1984a. Lós Ápidos de la Sierra de Guadarrama II. Familias Andrenidae y Megachilidae (Hymenoptera, Apoidea). Graellsia, 39 [1983]: 103126.

Pérez-ÍñIgo, C., 1984b. Los Ápidos de la Sierra de Guadarrama III. Familias Colletidae y Haliciidae [sic!] (Hymenoptera, Apoidea). Graellsia, 40: 129-157.
Rivas-Martínez, S. \& LoIDI, J., 1999. Biogeography of the Iberian Peninsula. Itinera Geobotanica, 13: 49-67.

Romera, L., 2004. La familia Geometridae Leach, 1815 en los bosques de Quercus de la Comunidad de Madrid (Insecta, Lepidoptera). Tesis Doctoral. Universidad Autónoma de Madrid. Madrid. 535 pp.

Romera, L., Cifuentes, J., Viejo, J. L. \& Fernández, J., 2002. Los geométridos del piso supramediterráneo de la Sierra de Guadarrama: estacionalidad y relación con las formaciones vegetales (Insecta: Lepidoptera: Geometridae). Boletín de la Asociación española de Entomología, 26(1-2): 145-162.

Viejo, J. L. \& Templado, J., 1986. Los piéridos, satíridos y ninfálidos (Lep.) de la región de Madrid en relación con las formaciones vegetales. Graellsia, 42: 237-265.

WAlter, H. \& LieTh, H., 1960. Klimadiagramm Weltatlas. Gustav Fischer Verlag. Jena.

WARNCKE, K., 1972. Beitrag zur Systematik und Verbreitung der Bienengattung Prosopis F. in der Westpaläarktis (Hymenoptera, Apoidea, Colletidae). Bulletin des Recherches agronomiques de Gembloux (N.S.), 5(3-4) [1970]: 745-768.

WARNCKE, K., 1978. Über die westpaläarktischen Arten der Bienengattung Colletes Latr. (Hymenoptera, Apoidea). Polskie Pismo Entomologiczne, 48: 329370.

WARnCKE, K., 1981. Beitrag zur Bienenfauna des Iran 12. Die Gattung Prosopis F., mit Bemerkungen zu weiteren bekannten und umbekannten paläarktischen Arten. Bollettino del Museo Civico di Storia naturale di Venezia, 31 [1980]: 145-195.

WARNCKE, K., 1992. 2. Beitrag zur Systematik und Verbreitung der Bienengattung Prosopis F. in der Westpaläarktis (Hym., Apidae). Linzer biologische Beiträge, 24(2): 747-801.
Recibido, 2-III-2005 Aceptado, 14-VI-2005 Publicado, 14-VII-2005 


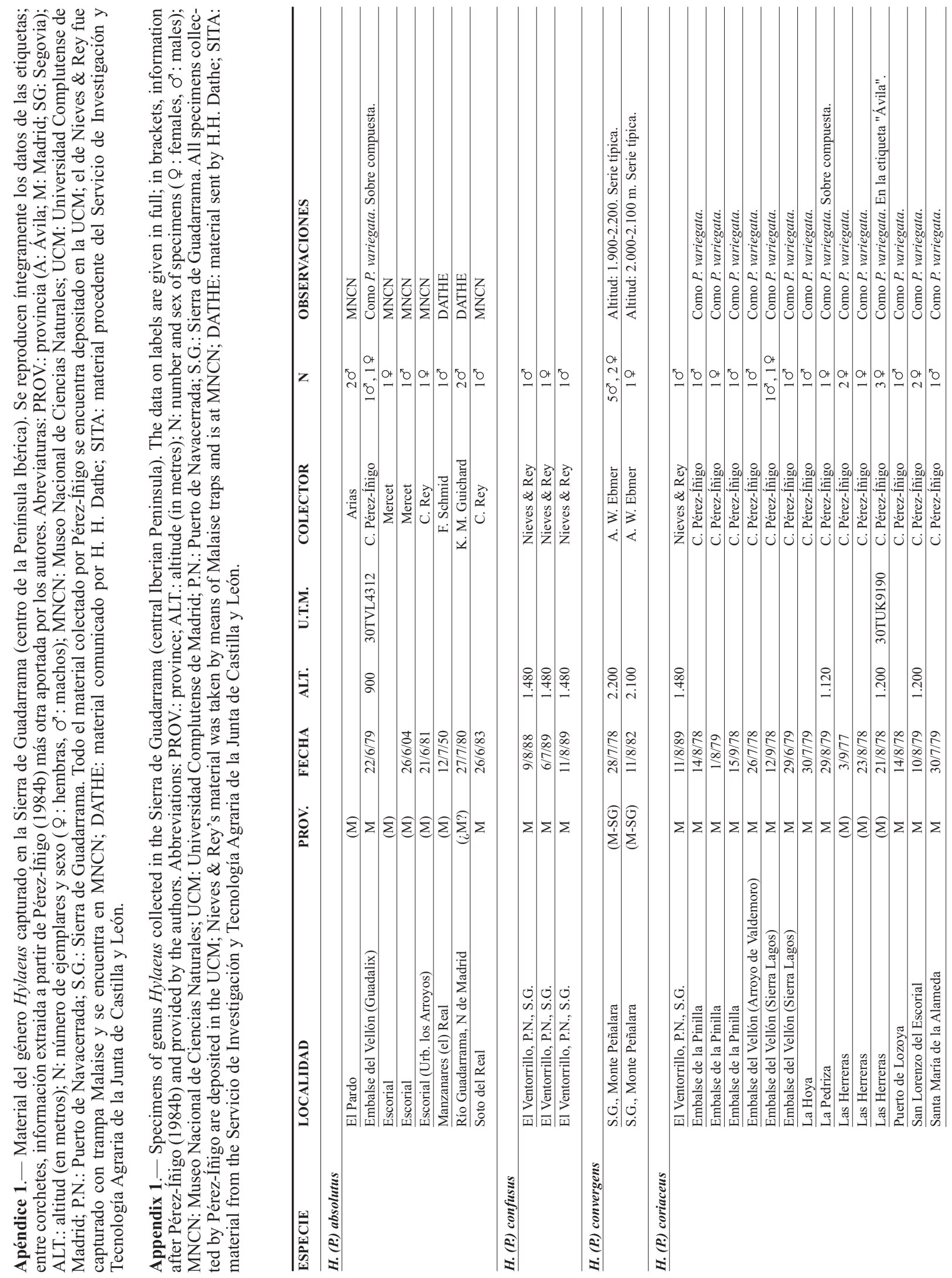




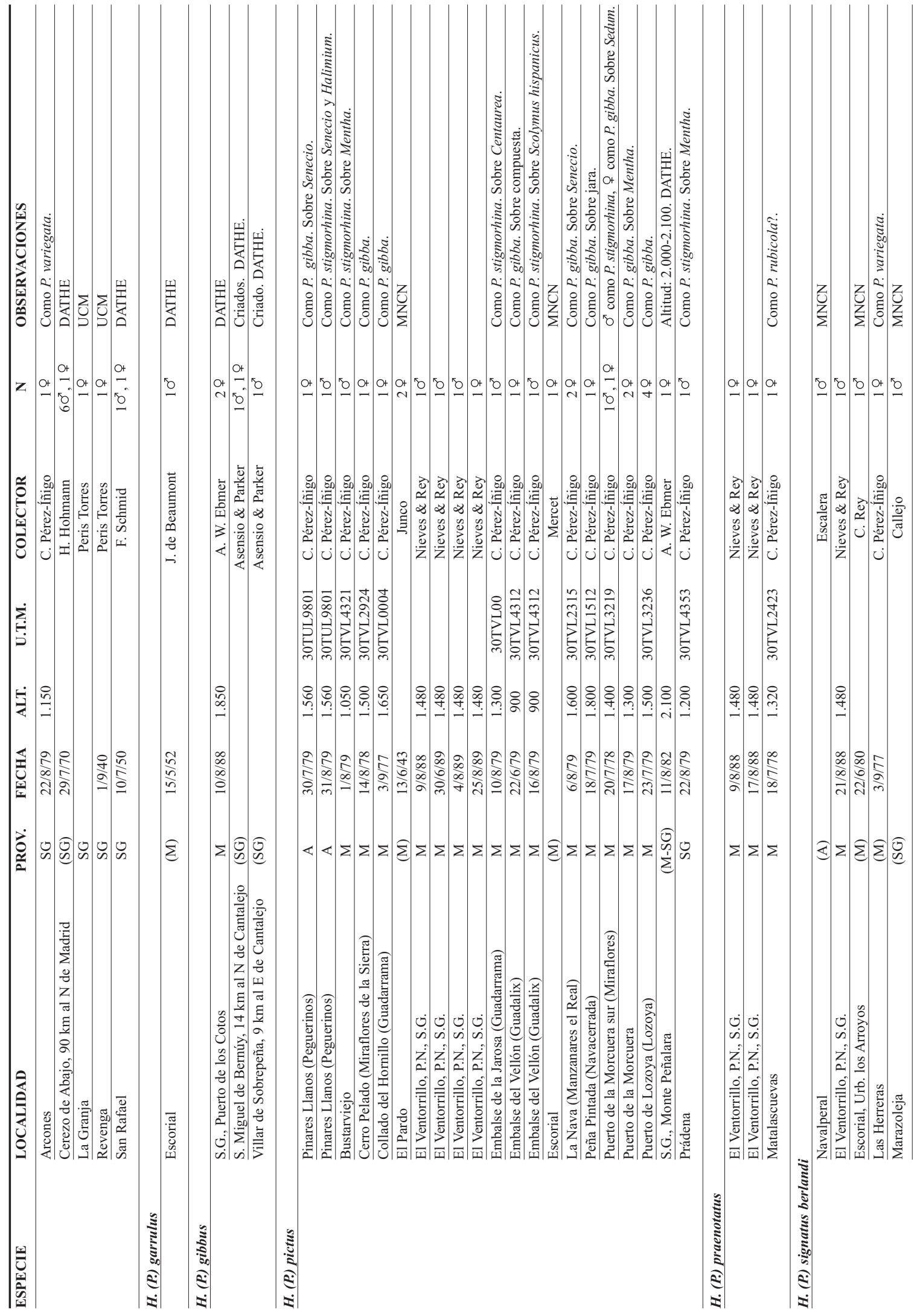



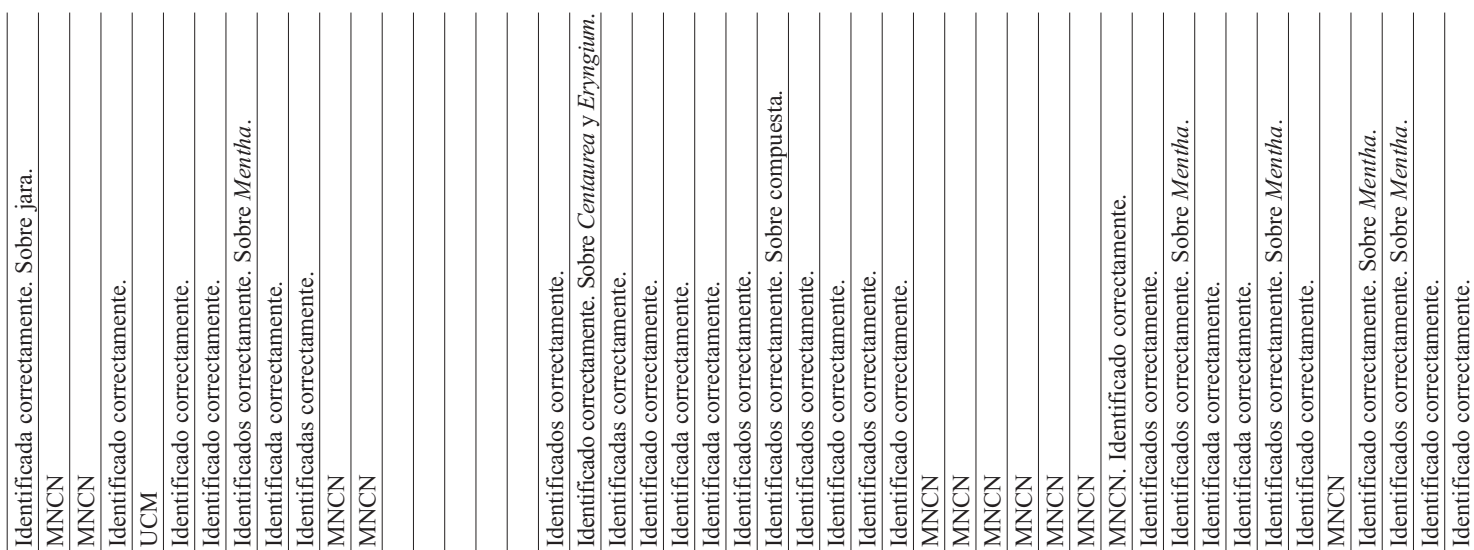

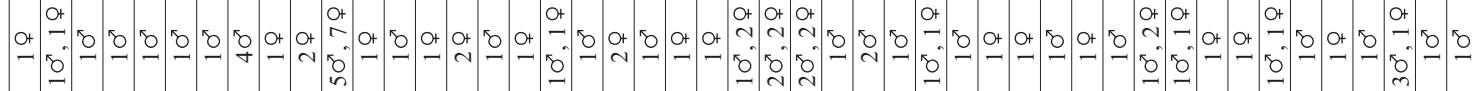

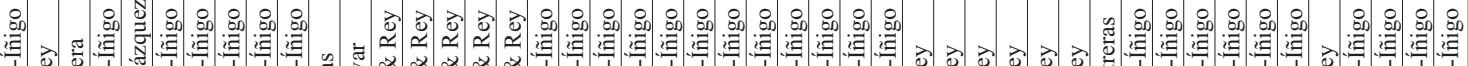

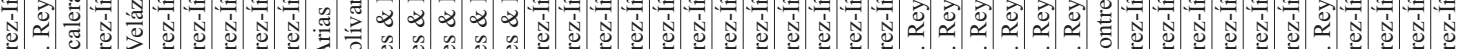
ن

๑

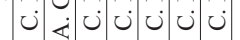

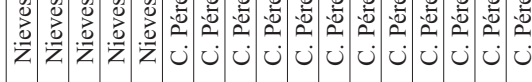

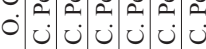
نे|نे⿺辶一

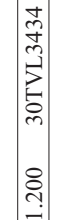

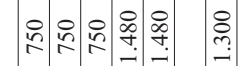

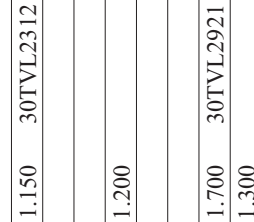

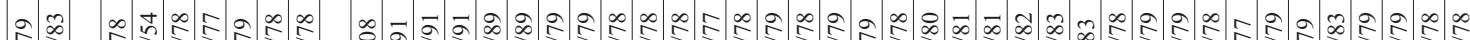

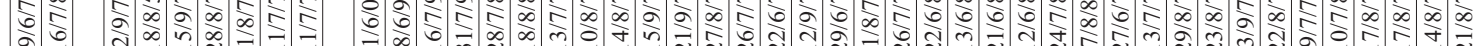

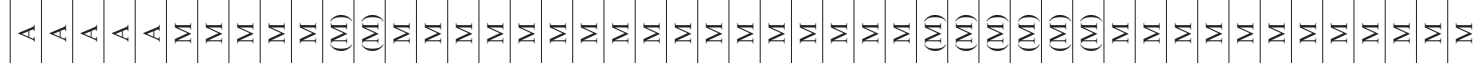

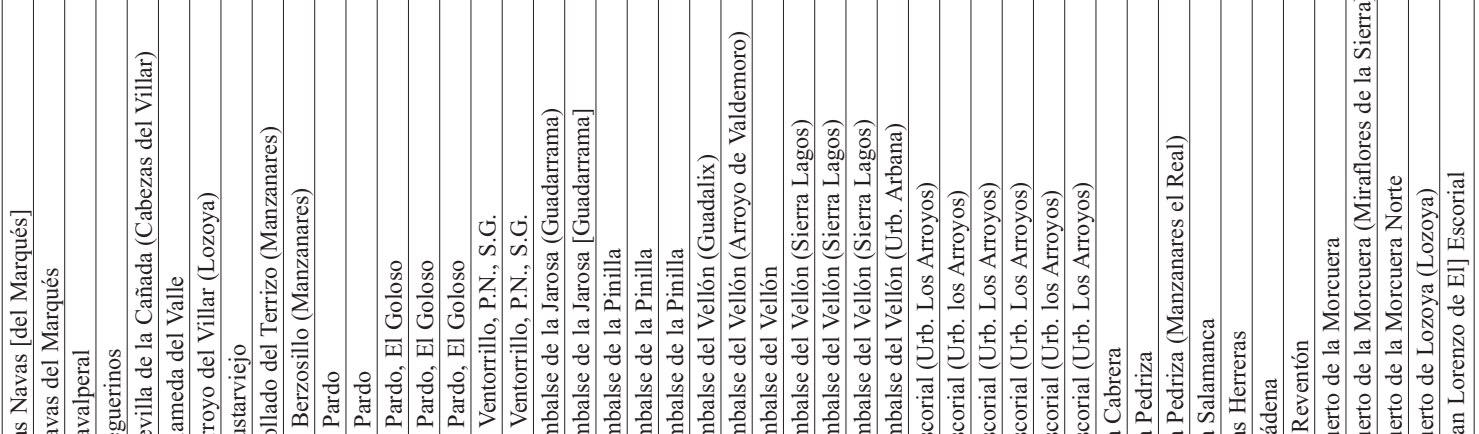




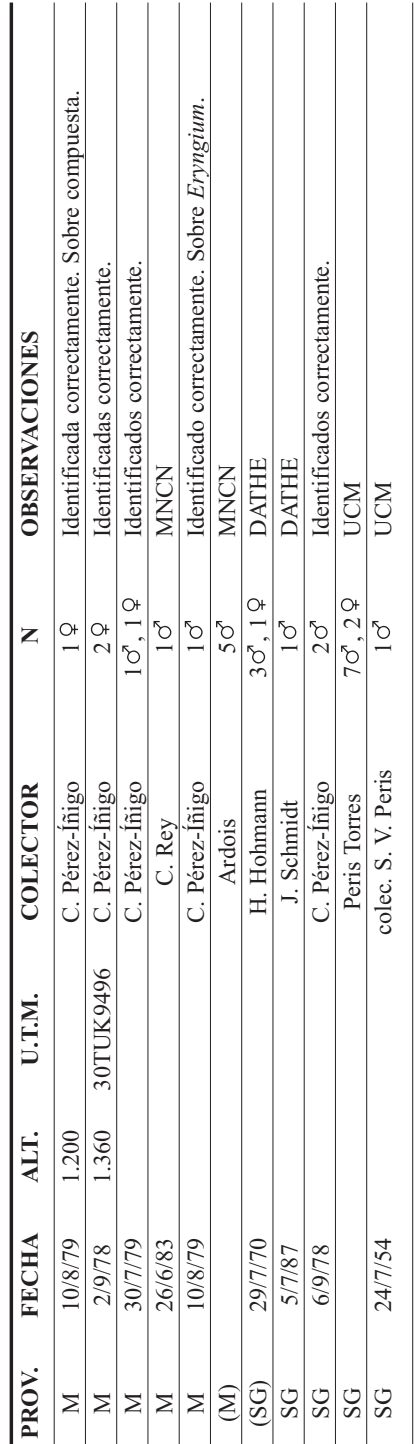

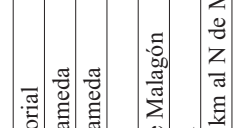

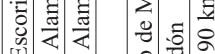

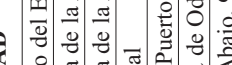

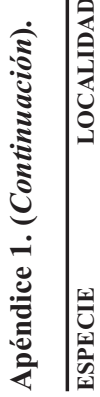

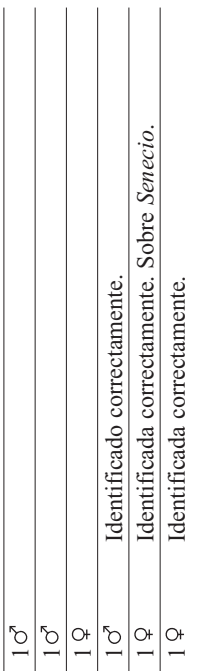

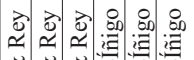

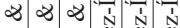

00

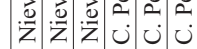

लै

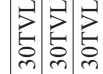

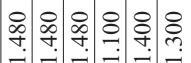

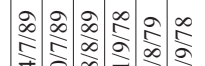

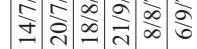

$\Sigma|\Sigma| \Sigma \mid \begin{array}{ll}0 \\ W\end{array}$

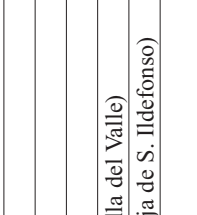

ن.

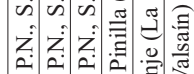

(1)

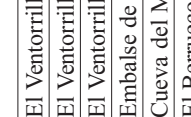

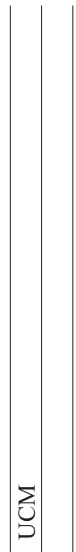

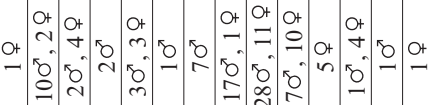

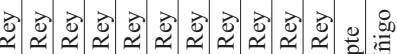
:

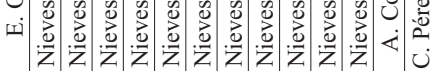

离

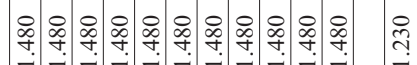

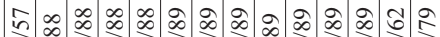

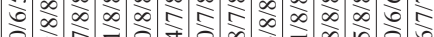

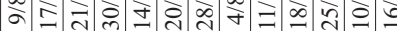

$\Sigma|\Sigma| \Sigma|\Sigma| \Sigma|\Sigma| \Sigma|\Sigma| \Sigma \mid v$

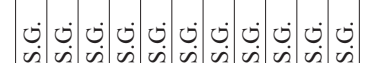

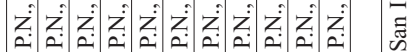

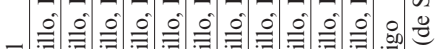

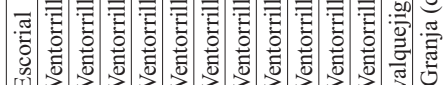

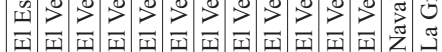

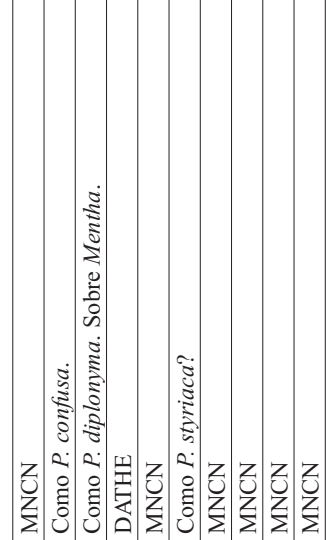

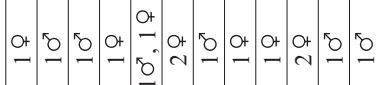

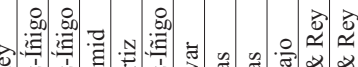

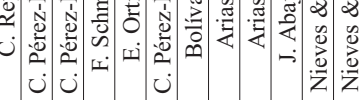

ำำ

空点

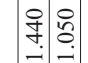

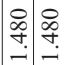

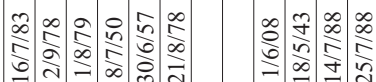

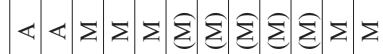

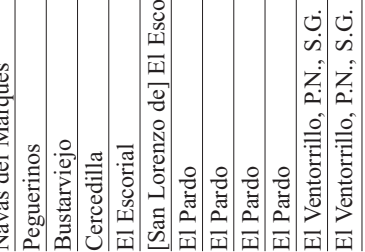

ะัะ 


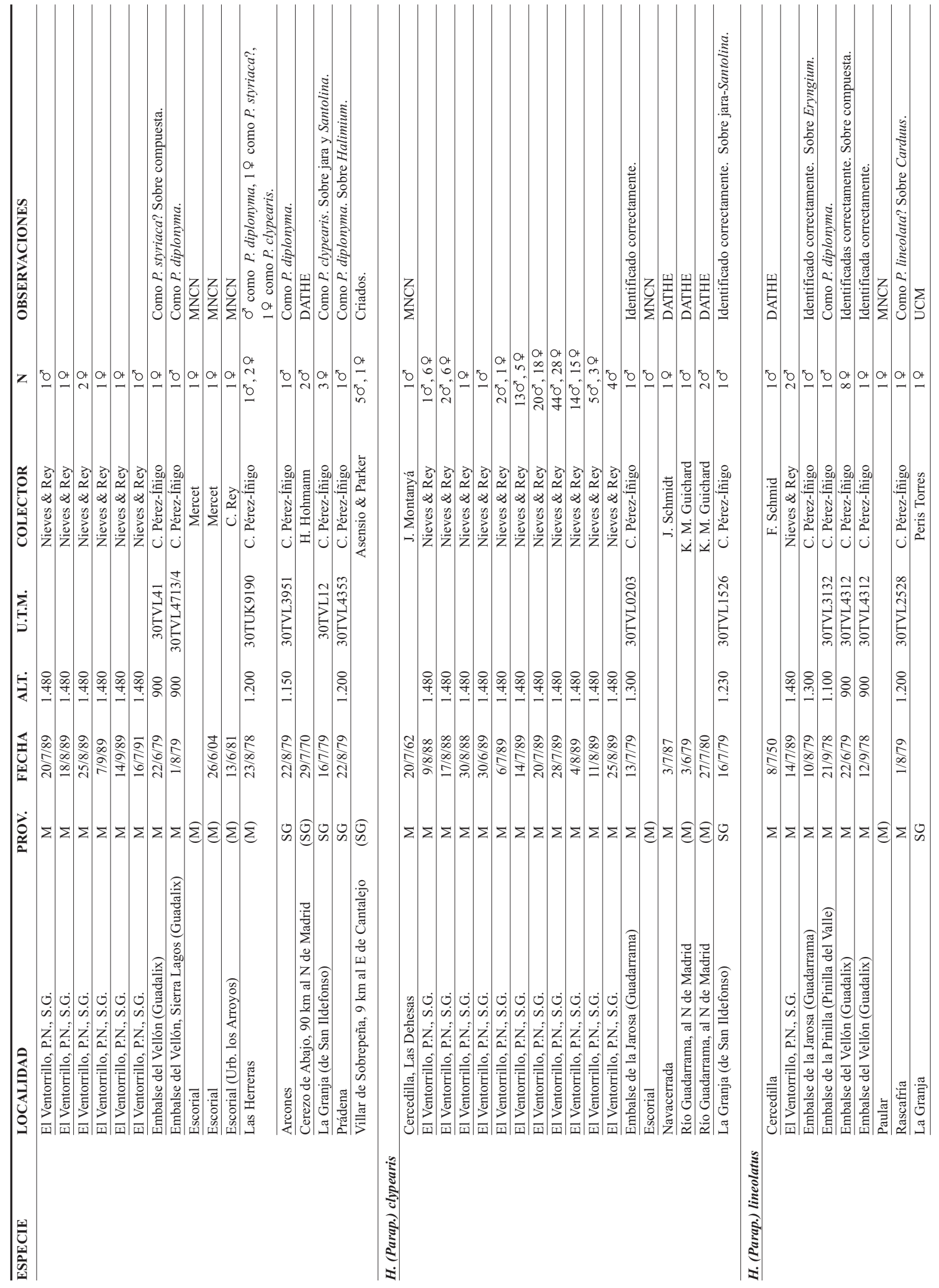




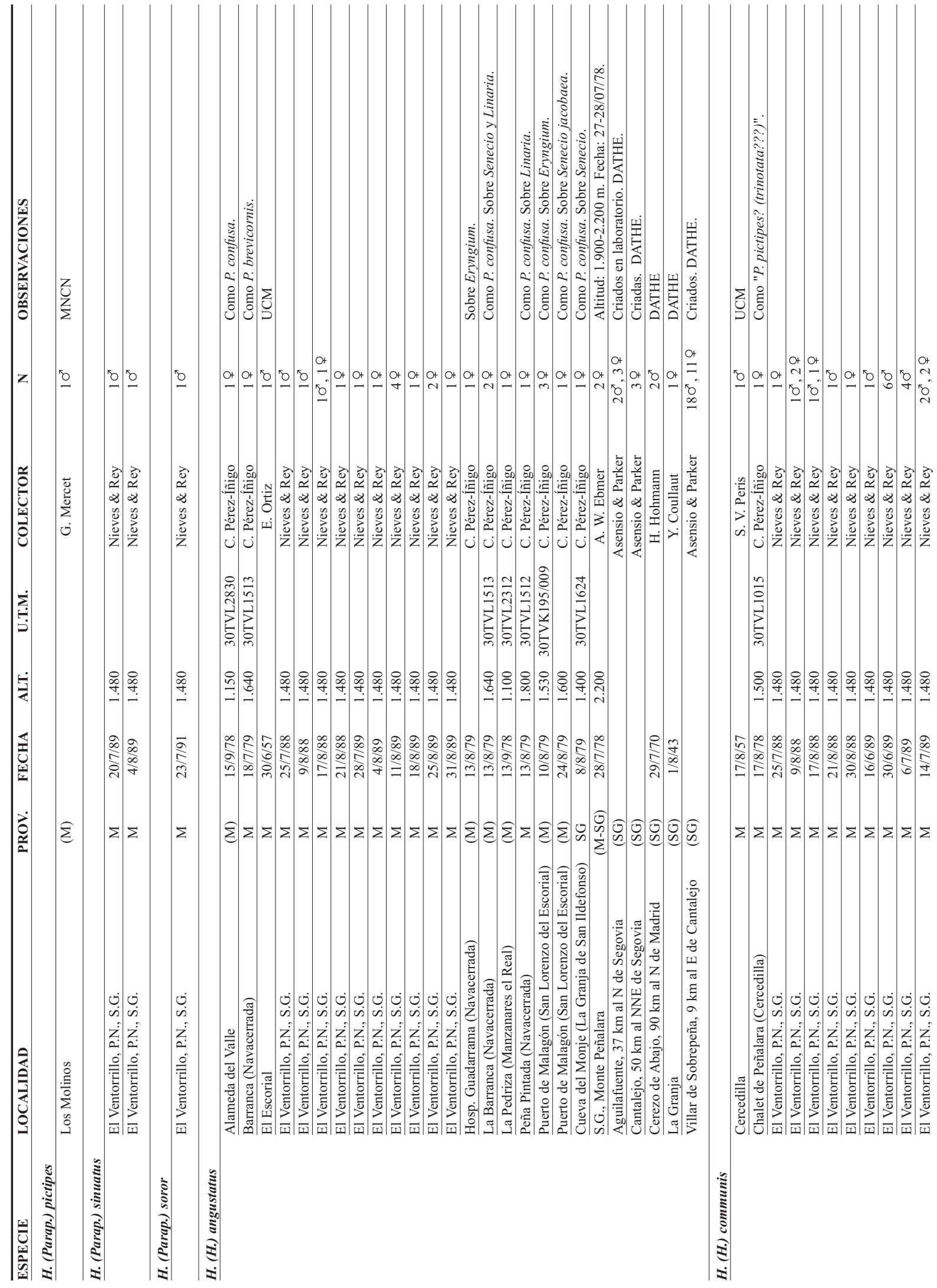




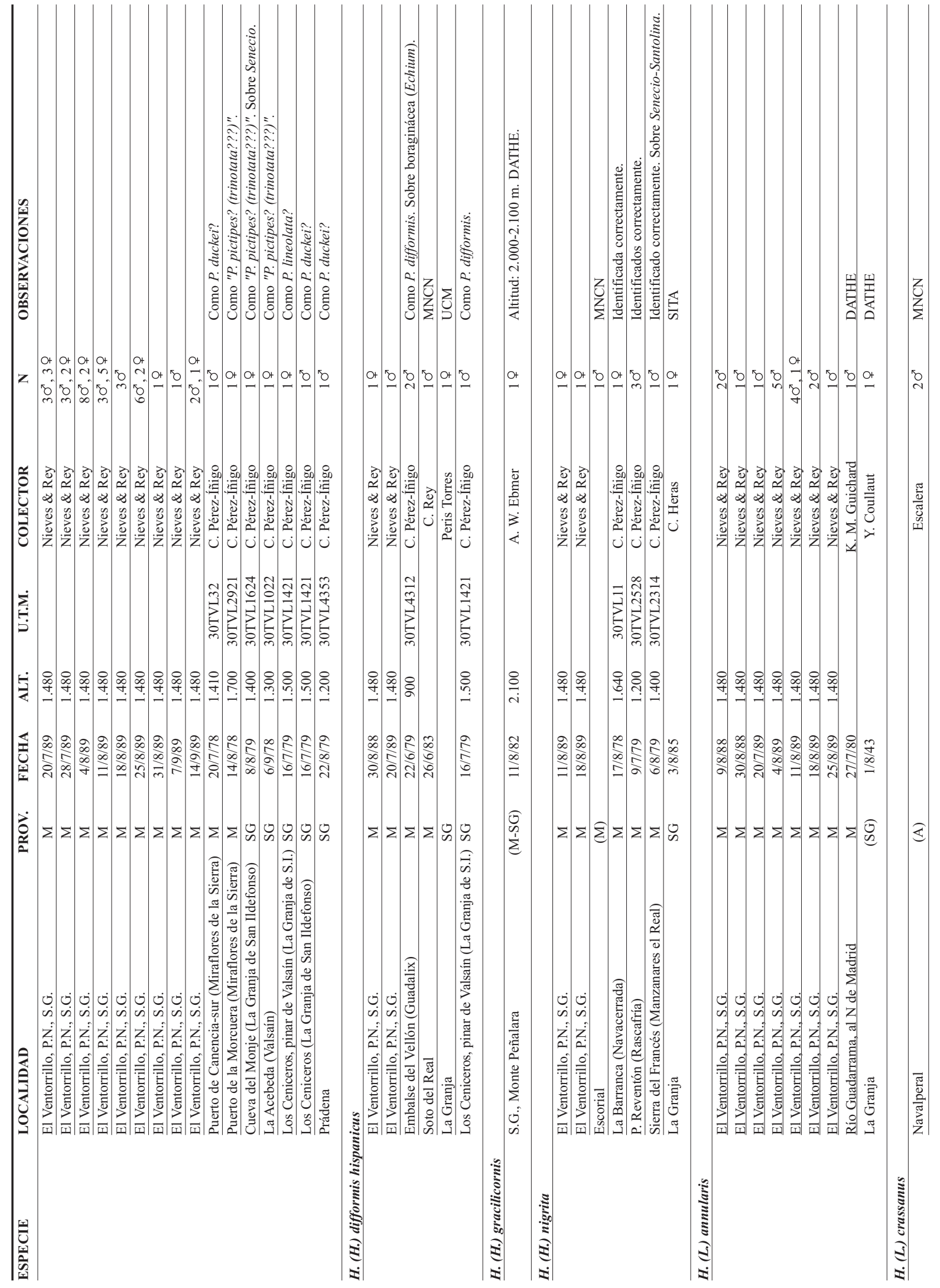




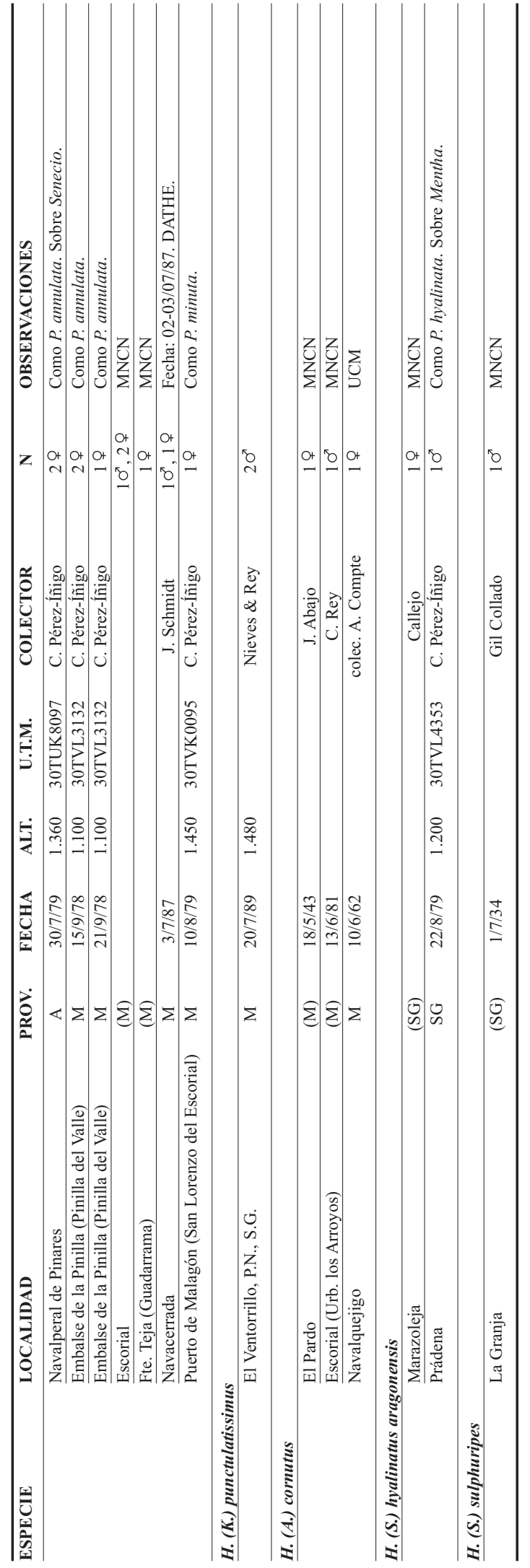




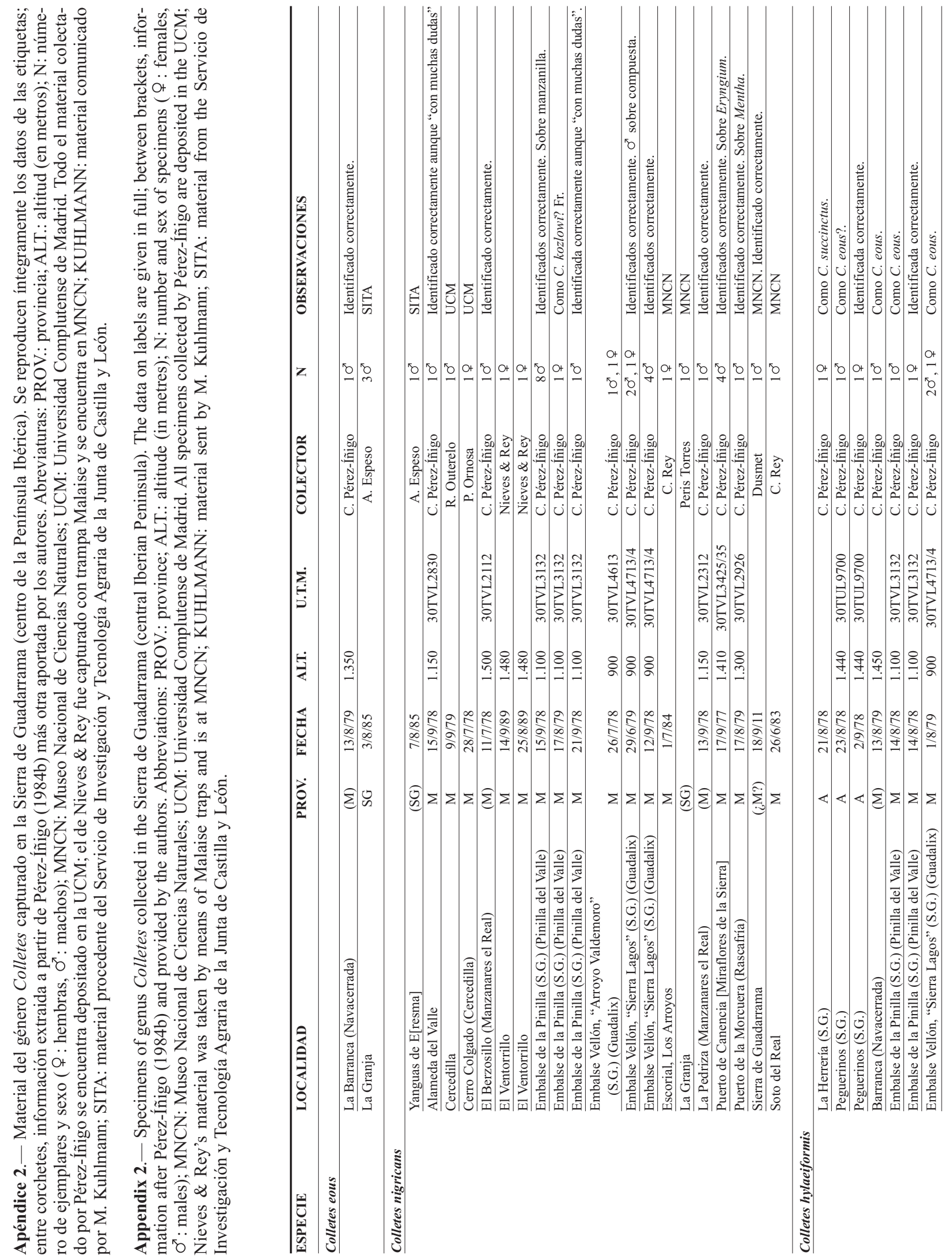




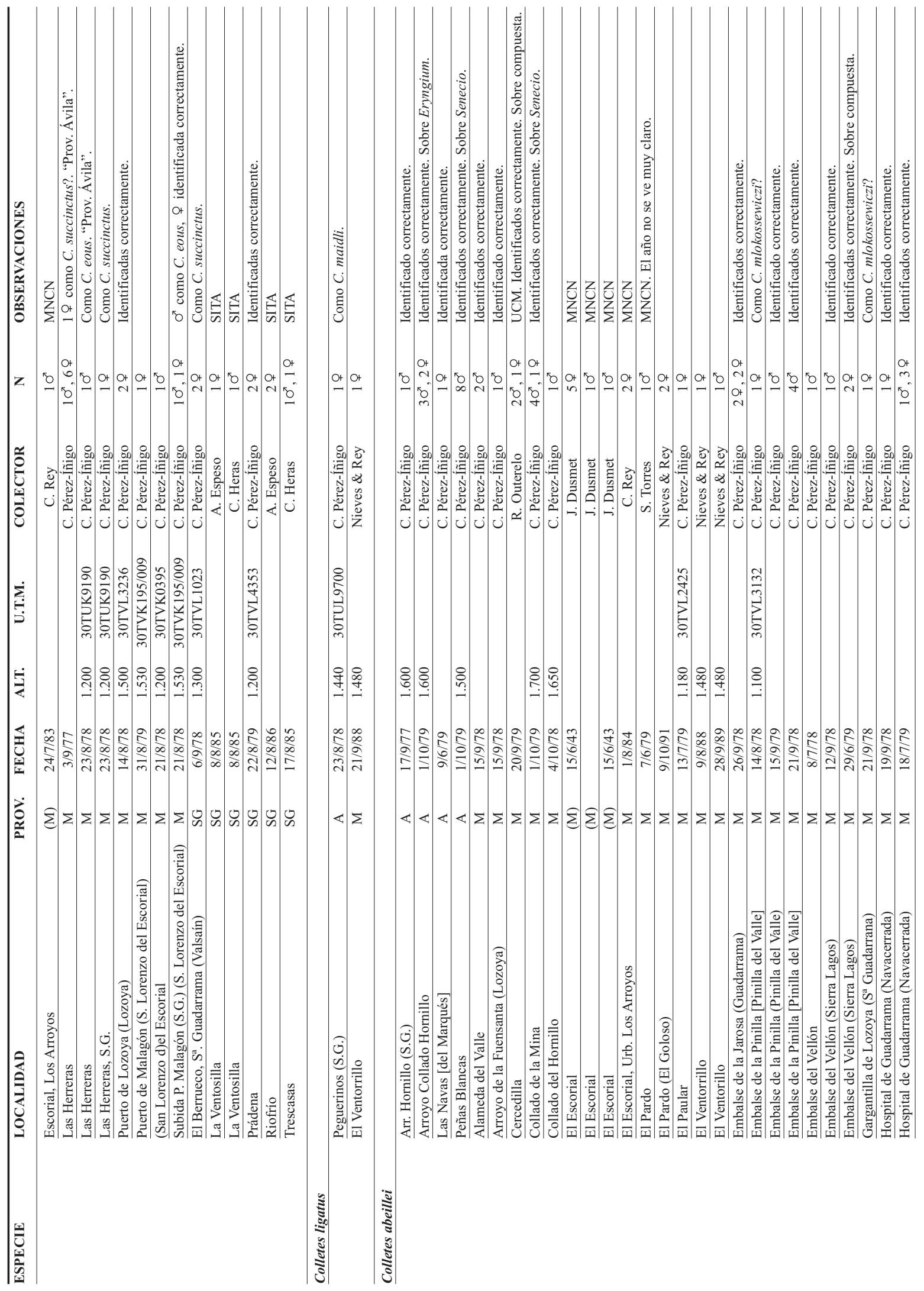




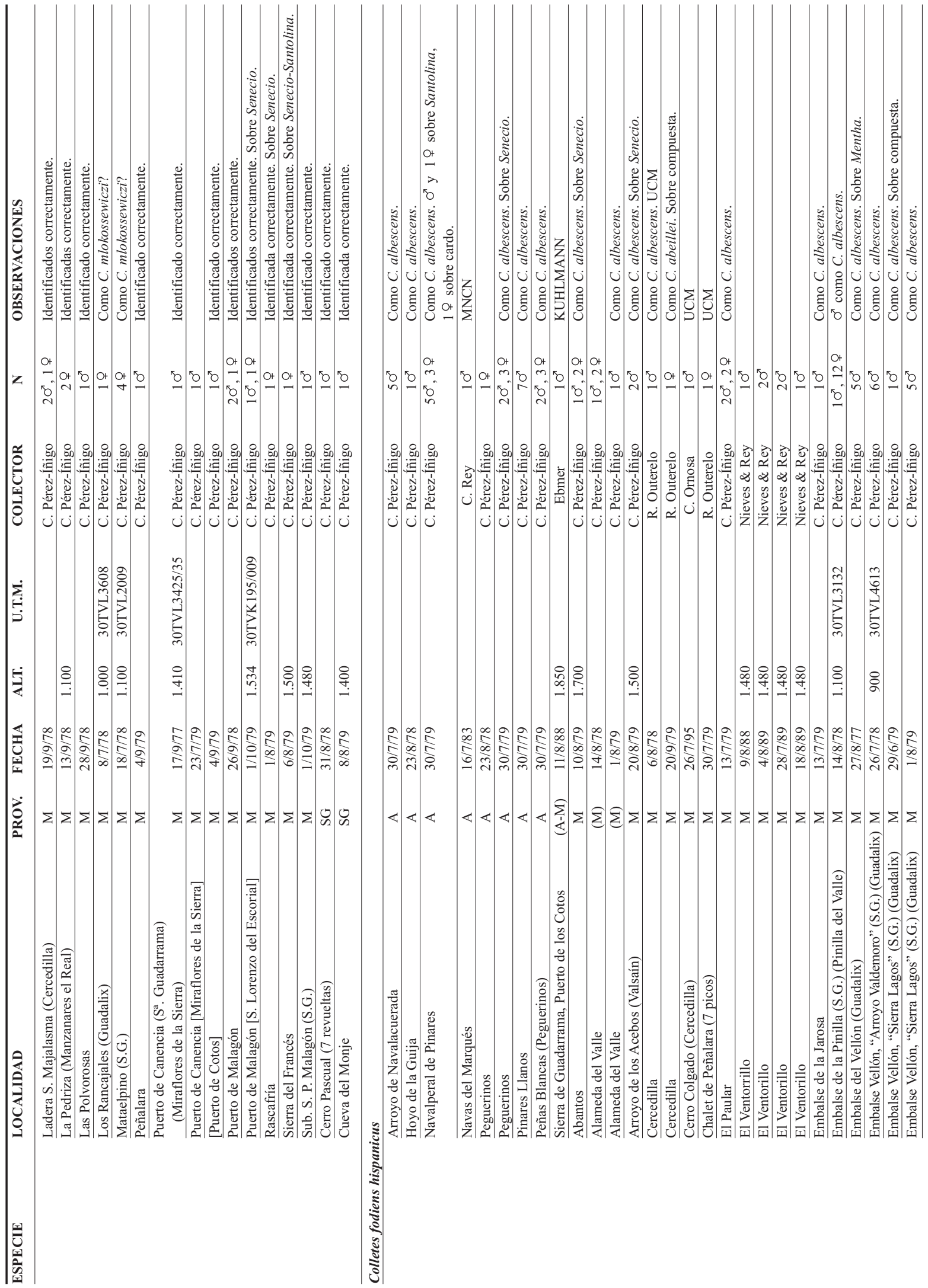




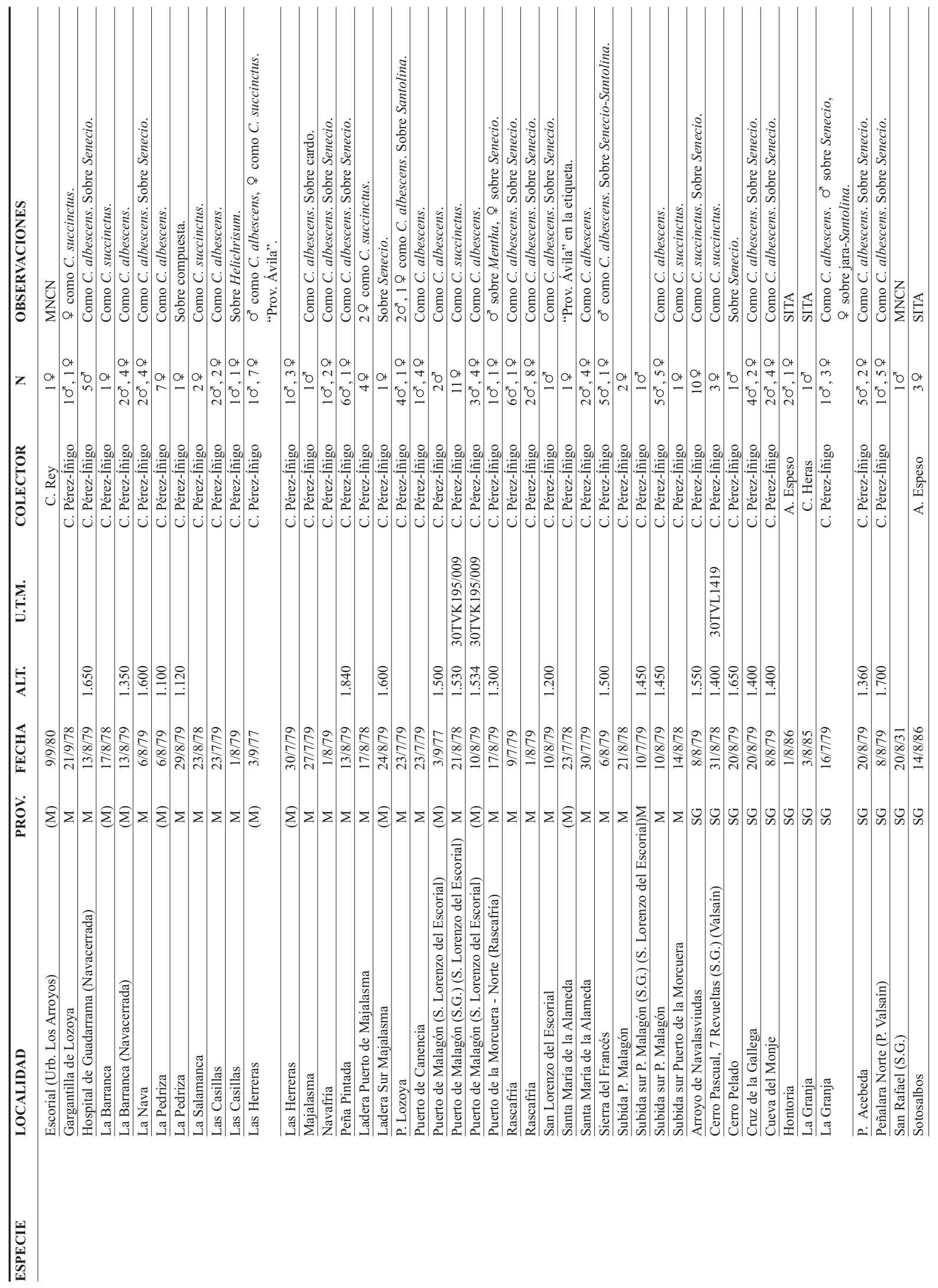




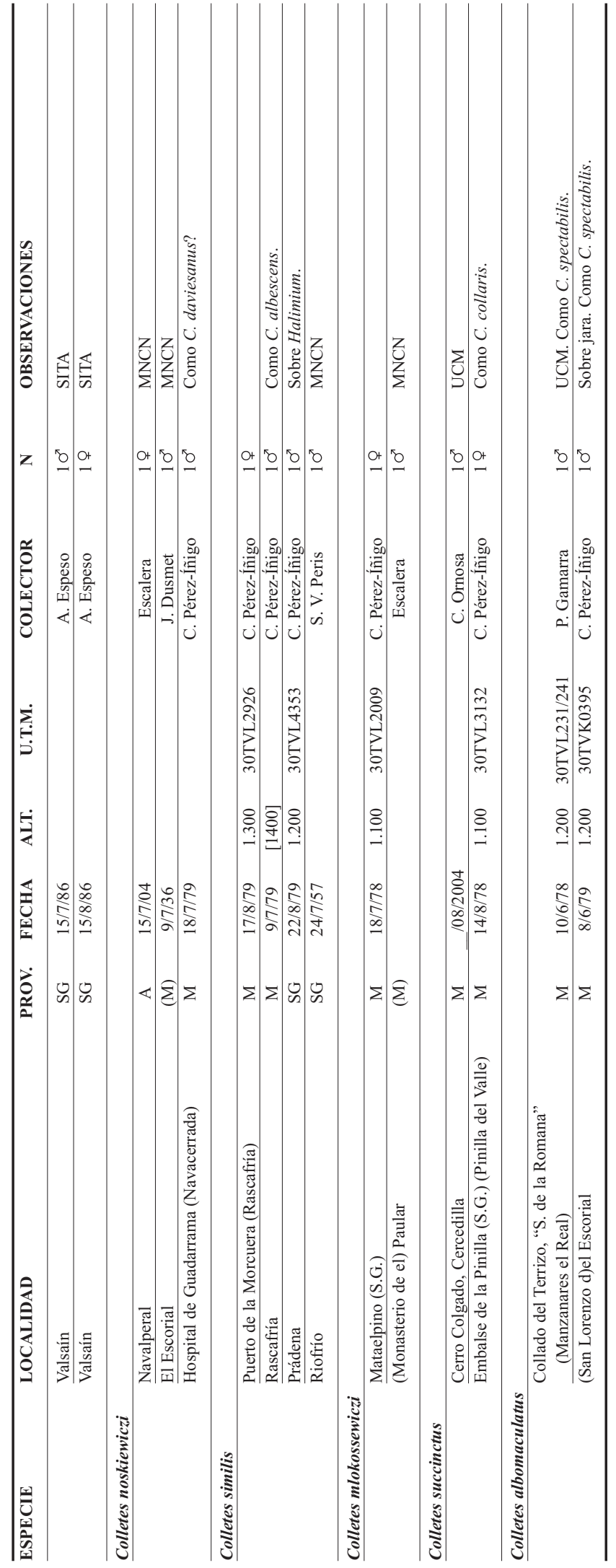

